\title{
P \\ Structural basis of nucleosome transcription mediated by Chd1 and FACT
}

\author{
Lucas Farnung $\odot$, Moritz Ochmann, Maik Engeholm and Patrick Cramer $₫$
}

\begin{abstract}
Efficient transcription of RNA polymerase II (PoI II) through nucleosomes requires the help of various factors. Here we show biochemically that Pol II transcription through a nucleosome is facilitated by the chromatin remodeler Chd1 and the histone chaperone FACT when the elongation factors Spt4/5 and TFIIS are present. We report cryo-EM structures of transcribing Saccharomyces cerevisiae Pol II-Spt4/5-nucleosome complexes with bound Chd1 or FACT. In the first structure, Pol II transcription exposes the proximal histone H2A-H2B dimer that is bound by Spt5. Pol II has also released the inhibitory DNA-binding region of Chd1 that is poised to pump DNA toward Pol II. In the second structure, Pol II has generated a partially unraveled nucleosome that binds FACT, which excludes Chd1 and Spt5. These results suggest that Pol II progression through a nucleosome activates Chd1, enables FACT binding and eventually triggers transfer of FACT together with histones to upstream DNA.
\end{abstract}

E ukaryotic transcription occurs within chromatin. This poses the long-standing question of how Pol II passes through a nucleosome, the fundamental unit of chromatin ${ }^{1,2}$ Biochemical $^{3-6}$ and single-molecule assays ${ }^{7,8}$ have shown that Pol II pauses at distinct positions within the proximal half of the nucleosome ${ }^{9-11}$. Pol II enters the nucleosome and stably pauses just before it reaches the nucleosome dyad, at superhelical location (SHL) -1 , both in vivo ${ }^{12}$ and in vitro ${ }^{3,6,13}$. To efficiently overcome the nucleosome barrier without loss of histones, Pol II requires the help of elongation factors ${ }^{5,6,14-16}$, ATP-dependent chromatin remodeling factors $^{17,18}$ and histone chaperones ${ }^{15,19,20}$.

Recently, structures of Pol II-nucleosome complexes ${ }^{5,6,13}$ have provided a starting point to elucidate the mechanisms of chromatin transcription. These structures showed that transcription into the nucleosome results in partial DNA unwrapping. However, available structures lack the factors that are critical for nucleosome transcription, particularly the chromatin remodeling factor Chd 1 (ref. ${ }^{21}$ ) and the histone chaperone FACT $^{22}$, a heterodimer of subunits Spt16 and Pob3. Chd1 and FACT form a complex ${ }^{23,24}$ and act in concert ${ }^{25}$. Binary structures of Chd1 (ref. ${ }^{26}$ ) or FACT ${ }^{27}$ bound to nucleosomes have revealed how these factors can recognize nucleosome substrates. Here we use a structure-function analysis to investigate how Chd1 and FACT facilitate Pol II passage through a nucleosome and arrive at a mechanistic model of factor-mediated nucleosome transcription.

\section{Results}

Chd1 and FACT promote nucleosome transcription. To understand how Chd1 and FACT facilitate nucleosome passage by Pol II, we reconstituted factor-facilitated Pol II transcription through a nucleosome in vitro. We designed an extended nucleosome substrate for the biochemical and structural investigation of nucleosome transcription (Fig. 1a). The substrate consists of a single nucleosome, formed on a modified Widom 601 sequence $^{6}$, with a 40-base pair (bp) upstream DNA extension (Methods). The DNA extension has a 9-nucleotide (nt) $3^{\prime}$-overhang ${ }^{5}$ that enables annealing of a fluorescently labeled RNA oligonucleotide and allows for Pol II binding and catalytic RNA extension upon addition of nucleoside triphosphates (NTPs).
We then used a fluorescence-based RNA extension assay to determine the efficiency of nucleosome transcription in the presence of S. cerevisiae Pol II and various factors (Fig. $1 \mathrm{~b}$ and Extended Data Fig. 1a). We formed a Pol II elongation complex on the extended nucleosome substrate, provided the elongation factors Spt4/5 (Spt 4 and Spt 5) and TFIIS, and initiated RNA elongation by the addition of $1 \mathrm{mM}$ NTPs. Samples were removed at specific time points, and the RNA products were separated by denaturing gel electrophoresis and quantified. We observed that Pol II paused when its leading edge was located at SHL -5 (Pol II active site at bp 1 of the nucleosome) and again when reaching SHL -1 (Pol II active site at bp 42) (Fig. 1b). These pausing positions correspond to previously described sites where Pol II pauses during nucleosome passage ${ }^{6,13}$. A small fraction of Pol II could overcome these barriers and transcribe trough the nucleosome, consistent with published observations ${ }^{3,16}$.

When Chd1 or FACT was added to the reactions containing Pol II, Spt $4 / 5$ and TFIIS, we observed a 5- or 7-fold increase in full-length product formation, respectively (Fig. 1b,c). The strong increase in full-length product in the presence of only Chd1 was dependent on the ATPase activity of Chd1, but a weak stimulatory effect was observed also with a catalytically inactive Chd1 variant (Fig. $1 \mathrm{~d}$ and Extended Data Fig. 1b). Indeed, Chd1 binding to a nucleosome may facilitate transcription because it leads to detachment of two turns of DNA at the Pol II entry site ${ }^{26}$. The stimulatory effects of Chd1 or FACT depend on the presence of Spt4/5 (Extended Data Fig. 1c,d). When both Chd1 and FACT were included, full-length RNA product formation increased only slightly (Fig. 1b,c), suggesting that the stimulatory effects of Chd1 and FACT are not additive.

Structure of Pol II-Spt4/5-nucleosome-Chd1 complex. To investigate the structural basis for Chd1 and FACT function during Pol II nucleosome passage, we formed a Pol II-Spt4/5-nucleosome complex in the presence of Chd1, FACT and the transition-state analog $\mathrm{ADP}-\mathrm{BeF}_{3}$ (Extended Data Fig. 2a-d). Transcription was carried out in the presence of GTP, CTP and UTP, and the complex was purified by size exclusion chromatography followed by mild crosslinking with glutaraldehyde (Methods). We prepared cryo-EM grids, collected a total of 3.76 million particles and obtained a reconstruction at a nominal resolution of $2.9 \AA$ (Table 1, Fig. 2, Extended 

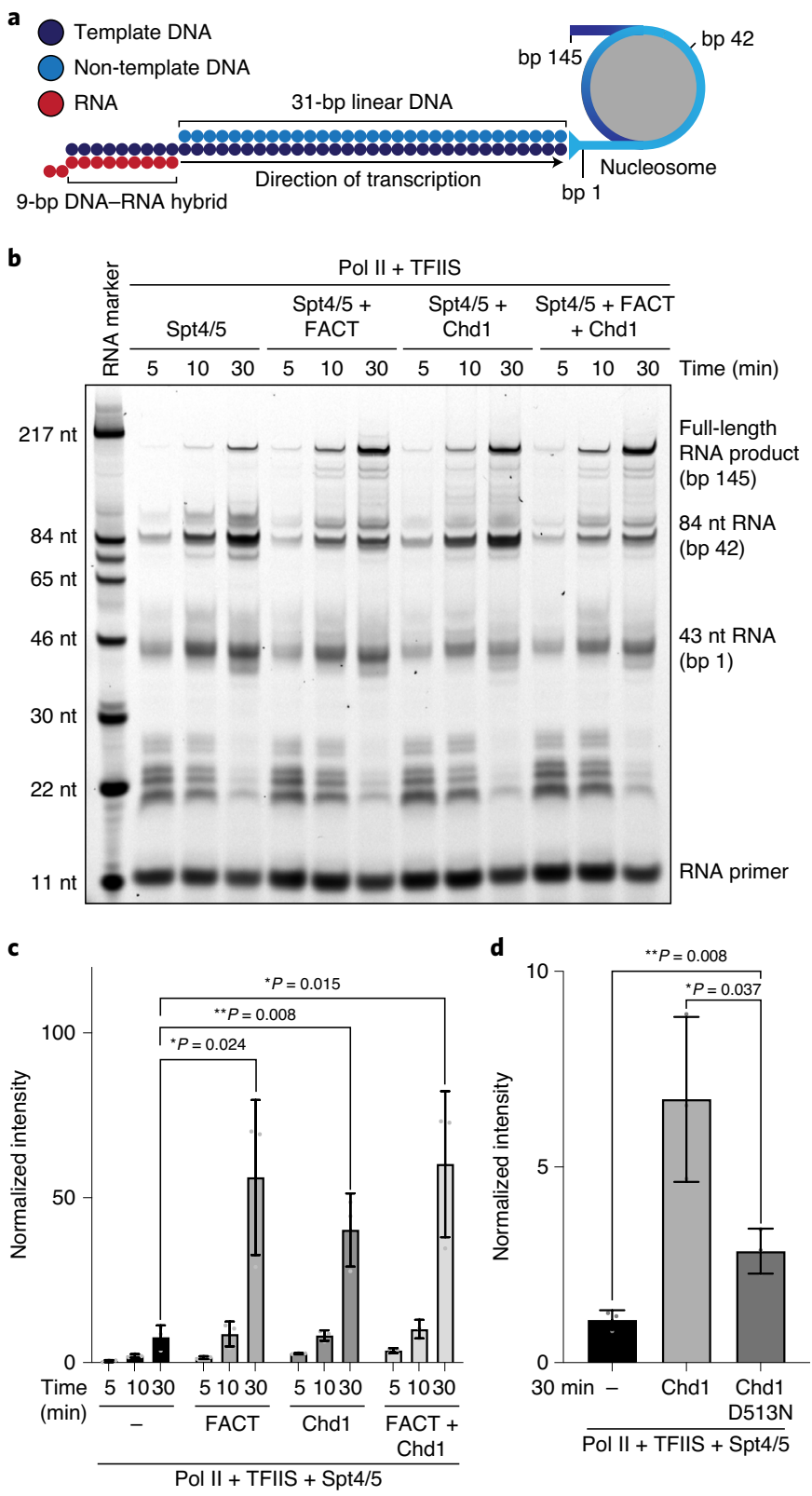

Fig. 1 | Chd1 and FACT stimulate nucleosome transcription. a, Schematic of nucleosome substrate used for formation of Pol II-nucleosome complexes and RNA extension assays. b, Nucleosome transcription assay shows an increase in full-length product in the presence of FACT, Chd1, or FACT and Chd1. RNA length and corresponding nucleosomal base pairs are indicated. c, Bar graph shows a significant increase in full-length product upon addition of FACT, Chd1, or FACT and Chd1 to Pol II-Spt4/5-TFIIS complexes after 30 min of transcription. $n=3$ independent experiments with ${ }^{\star} P<0.05$, ${ }^{\star \star} P<0.01$ with two-tailed $t$ test. d, Mutation of Chd1 to eliminate ATPase activity (D513N) strongly decreases production of full-length RNA product during nucleosomal transcription. $n=3$ independent experiments with ${ }^{\star} P<0.05$, ${ }^{\star \star} P<0.01$ with two-tailed $t$ test. Error bars represent \pm standard deviation. Unprocessed gel images and derived values for plots are provided as source data and in Supplementary Data 1.

Data Fig. 3 and Supplementary Video 1). We placed known structures and homology models of Pol $\mathrm{II}^{28}$, Spt4/5 (ref. ${ }^{29}$ ) and the nucleosome core particle ${ }^{30}$ into the density, adjusted them and modeled the remaining DNA (Extended Data Fig. 4). Additional density was observed for Chd1, but not for FACT, and was fitted
Table 1 | Cryo-EM data collection, refinement and validation statistics

\begin{tabular}{|c|c|c|}
\hline & $\begin{array}{l}\text { Pol II-Spt4/5- } \\
\text { nucleosome-Chd1 } \\
\text { structure (EMD- } \\
12449 \text {, PDB 7NKX) }\end{array}$ & $\begin{array}{l}\text { Pol II-Spt4/5- } \\
\text { nucleosome-FACT } \\
\text { structure (EMD- } \\
12450, \text { PDB 7NKY) }\end{array}$ \\
\hline \multicolumn{3}{|l|}{ Data collection and processing } \\
\hline Magnification & 81,000 & 81,000 \\
\hline Voltage (kV) & 300 & 300 \\
\hline Electron exposure $\left(\mathrm{e}-/ \AA^{2}\right)$ & 39.87 & 40.25 \\
\hline Defocus range $(\mu \mathrm{m})$ & $0.5-1$ & $0.5-1.2$ \\
\hline Pixel size $(\AA)$ & 1.05 & 1.05 \\
\hline Symmetry imposed & $\mathrm{Cl}$ & $\mathrm{C} 1$ \\
\hline Initial particle images (no.) & $3,755,390$ & $5,227,093$ \\
\hline Final particle images (no.) & 30,876 & 47,138 \\
\hline Map resolution $(\AA)$ & 2.9 & 3.2 \\
\hline FSC threshold & 0.143 & 0.143 \\
\hline Map resolution range $(\AA)$ & $2.65-8.30$ & $2.95-11$ \\
\hline \multicolumn{3}{|l|}{ Refinement } \\
\hline $\begin{array}{l}\text { Initial model used } \\
\text { (PDB code) }\end{array}$ & $\begin{array}{l}3 \mathrm{LZO}, 509 \mathrm{G}, 3 \mathrm{PO} 2 \\
2 \mathrm{EXU}\end{array}$ & $\begin{array}{l}\text { 3LZO, 6UPL, 4IOY, } \\
4 \mathrm{PQ} 0,3 \mathrm{PO} 2\end{array}$ \\
\hline Model resolution $(\AA)$ & 3.3 & 3.4 \\
\hline FSC threshold & 0.5 & 0.5 \\
\hline Model resolution range $(\AA)$ & $2.7-8.30$ & 3-11 \\
\hline Map sharpening $B$ factor $\left(\AA^{2}\right)$ & $-38($ Map A) & -34.8 (Map 1) \\
\hline \multicolumn{3}{|l|}{ Model composition } \\
\hline Non-hydrogen atoms & 49,719 & 51,842 \\
\hline Protein residues & 5,506 & 5,734 \\
\hline Ligands & 11 & 10 \\
\hline \multicolumn{3}{|l|}{$B$ factors $\left(\AA^{2}\right)$} \\
\hline Protein & 100.03 & 125.18 \\
\hline Ligand & 101.47 & 131.60 \\
\hline \multicolumn{3}{|l|}{ R.m.s. deviations } \\
\hline Bond lengths $(\AA)$ & 0.008 & 0.006 \\
\hline Bond angles $\left({ }^{\circ}\right)$ & 1.174 & 0.973 \\
\hline \multicolumn{3}{|l|}{ Validation } \\
\hline MolProbity score & 1.76 & 1.70 \\
\hline Clashscore & 7.04 & 6.55 \\
\hline Poor rotamers (\%) & 0.10 & 0.02 \\
\hline \multicolumn{3}{|l|}{ Ramachandran plot } \\
\hline Favored (\%) & 94.49 & 95.13 \\
\hline Allowed (\%) & 5.51 & 4.87 \\
\hline Disallowed (\%) & 0.00 & 0.00 \\
\hline
\end{tabular}

with the structure of Chd 1 in its post-translocated state ${ }^{26}$ (Extended Data Figs. 4h,i and 5a). The structure was real-space refined and has good stereochemistry (Table 1).

The structure shows that Pol II adopts the active post-translocated state and has transcribed $27 \mathrm{bp}$ into the nucleosome, as observed biochemically (Extended Data Fig. 2c). The Pol II front edge and active site are located around SHL -3 and -4.5 , respectively (Fig. 2b,c and Extended Data Fig. 4a). At this stage, Pol II has unwrapped $45 \mathrm{bp}$ of nucleosomal DNA, exposing the proximal histone $\mathrm{H} 2 \mathrm{~A}-\mathrm{H} 2 \mathrm{~B}$ dimer (Extended Data Fig. 4a,g). Spt4/5 binds 
a
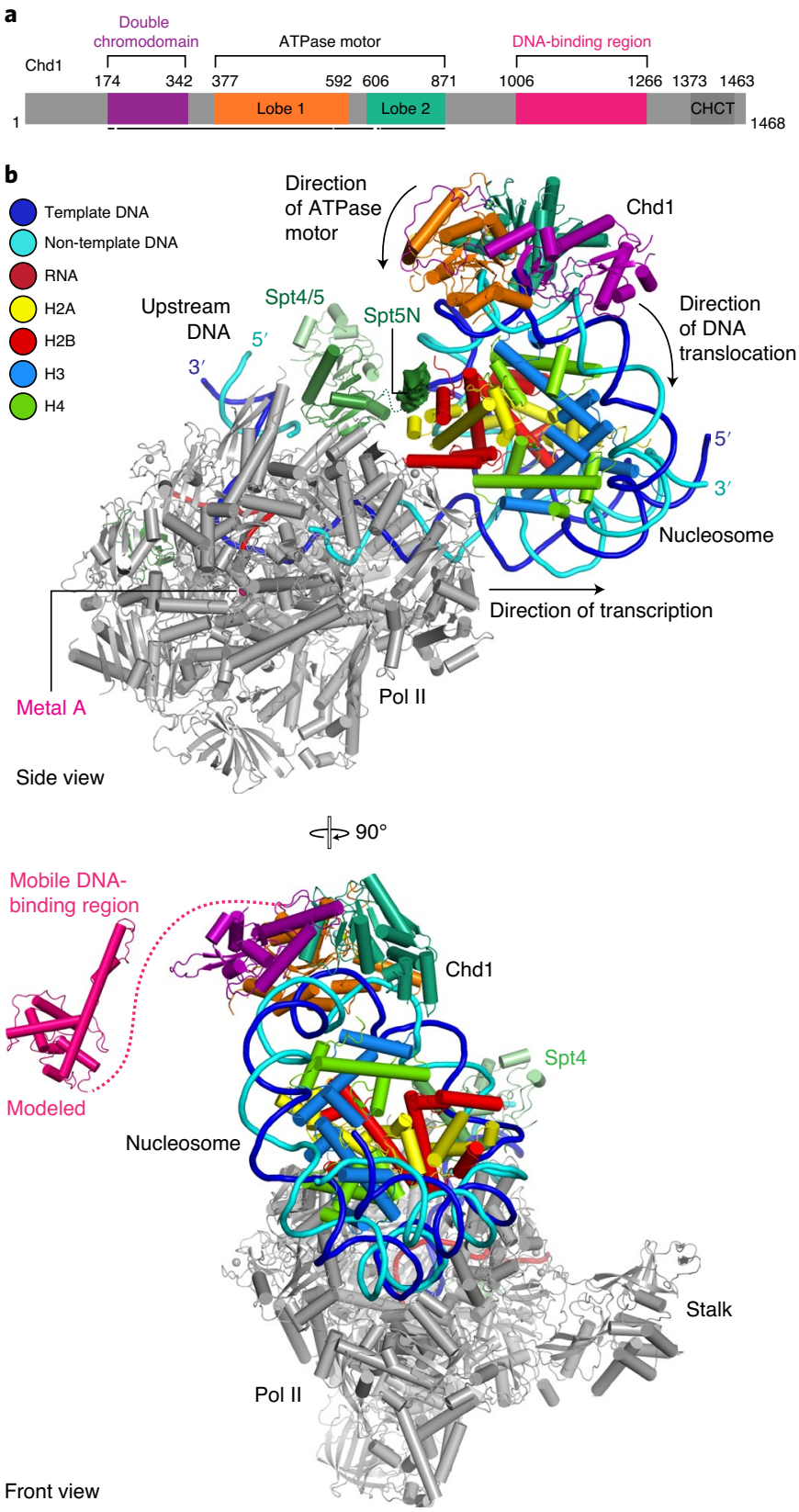

c

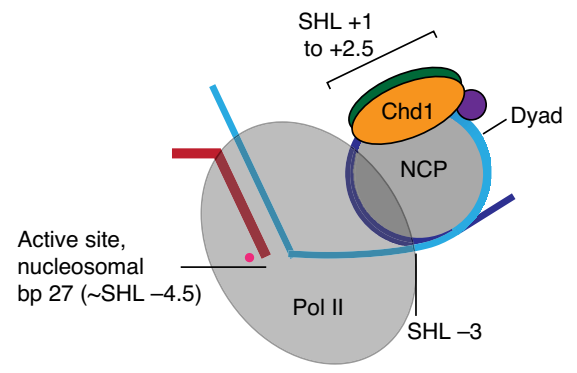

Fig. 2 | Pol ||-Spt4/5-nucleosome-Chd1 structure. a, Chd1 domain architecture. Residues at domain boundaries are indicated. Regions modeled in the Pol II-Spt4/5-nucleosome-Chd1 structure are indicated with a black bar. The same color coding is used throughout. b, Two views of the structure related by a $90^{\circ}$ rotation. The same color code for Pol II, Spt4/5, histones, metal A, RNA, and template and non-template DNA is used throughout. Spt5N density is shown in surface representation. c, Schematic of the structure indicating key elements.

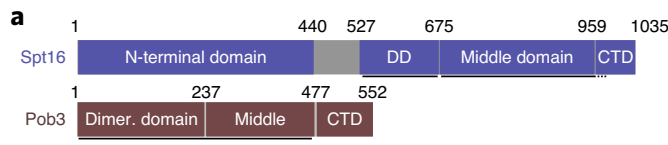

b
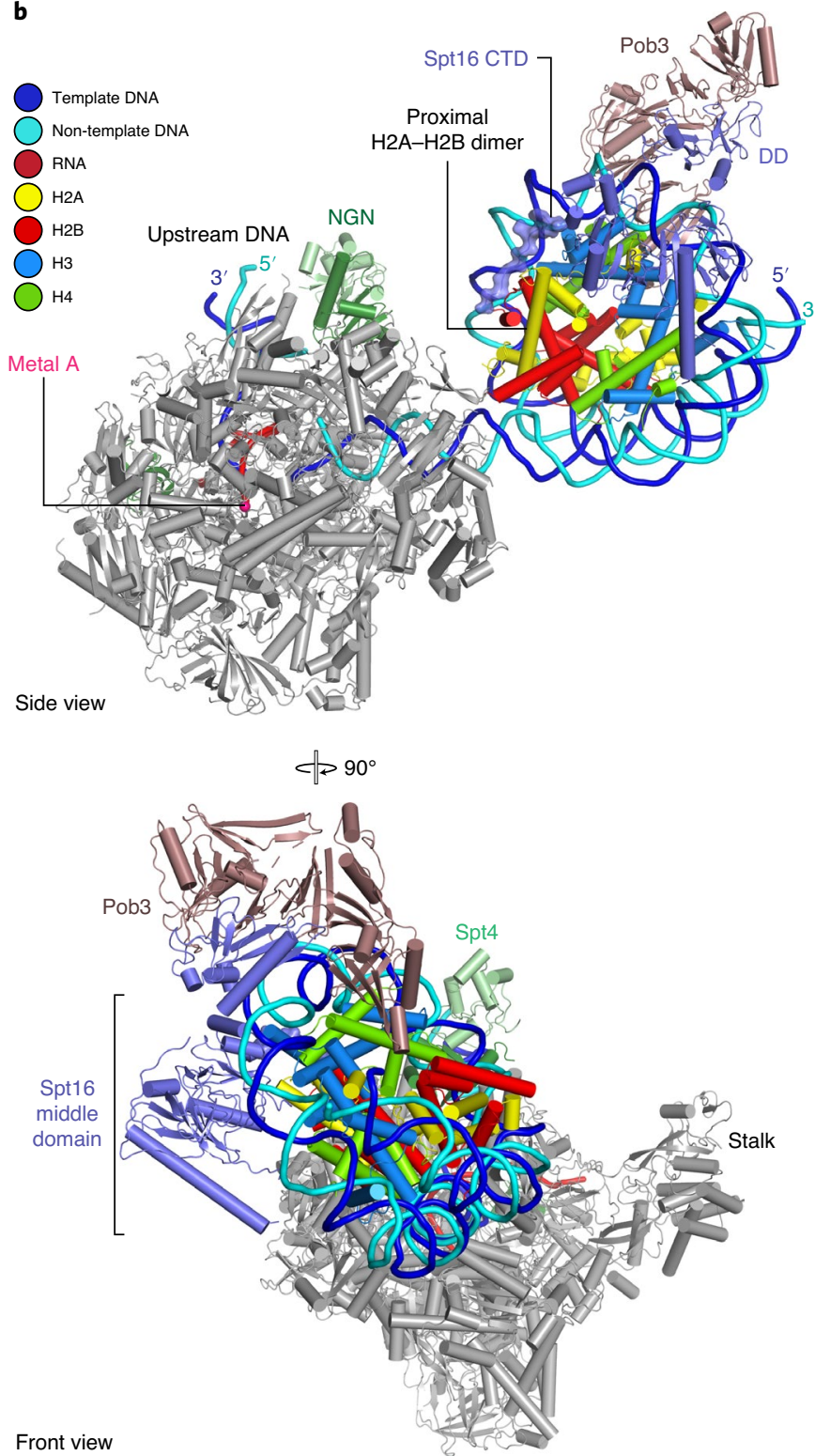

C

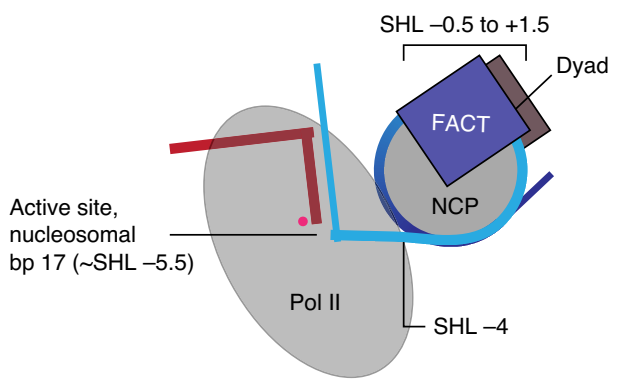

Fig. 3 | Pol II-Spt4/5-nucleosome-FACT structure. a, Domain architecture of FACT subunits Spt16 and Pob3 (DD, dimerization domain; CTD, C-terminal domain). Residues at domain boundaries are indicated. Regions modelled in the Pol II-Spt4/5-nucleosome-FACT structure are indicated with a black bar. $\mathbf{b}$, Two views of the structure related by a $90^{\circ}$ rotation. $\mathbf{c}$, Schematic of the structure indicating key elements. 


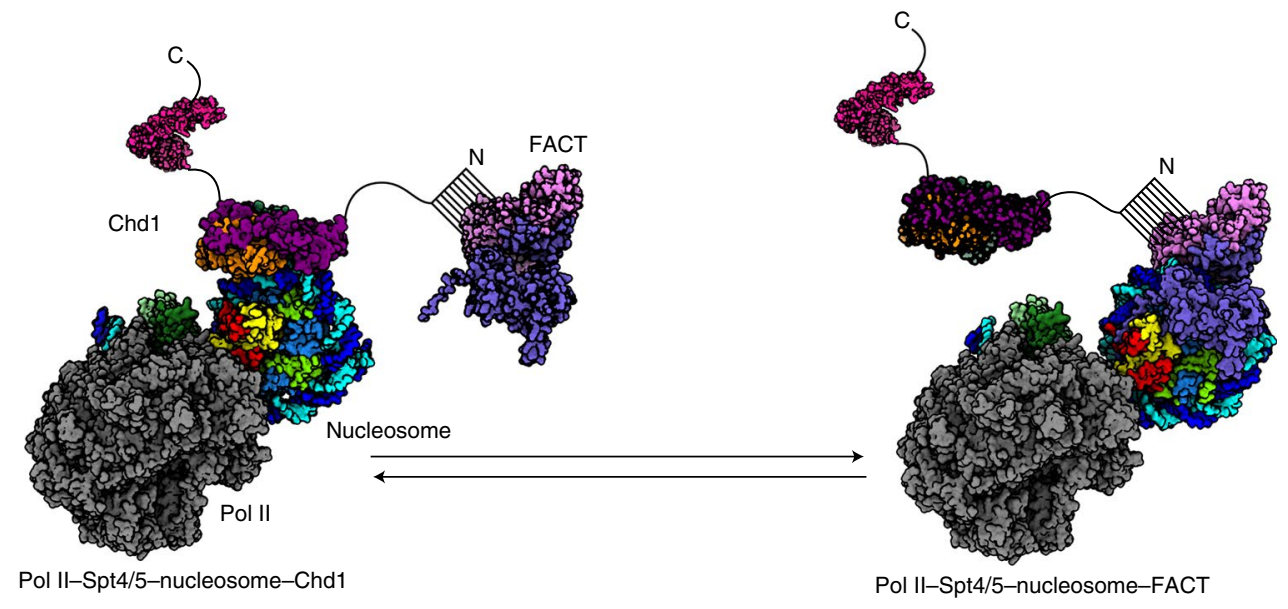

Fig. 4 | Model for Pol II passage through a nucleosome. a, Model for Pol II progression through the proximal part of a nucleosomal substrate. For details, please compare text.

around the Pol II cleft and the RNA exit channel, as previously observed $^{31,32}$. The NGN domain of Spt5 is sandwiched between upstream DNA that emerges from Pol II and the nucleosome located downstream. Some density was observed for the negatively charged N-terminal region of Spt5 (Spt5N). Spt5N interacts with the exposed proximal $\mathrm{H} 2 \mathrm{~A}-\mathrm{H} 2 \mathrm{~B}$ dimer, likely stabilizing it when DNA is absent (Fig. 2b and Extended Data Fig. 5b).

Chd1 activation and Pol II progression. The structure shows that Chd1 binds the partially unwrapped nucleosome. Chd1 uses its double chromodomain and its ATPase motor domain to contact the nucleosome at SHLs +1 and +2 , respectively (Fig. 2 and Extended Data Fig. 4a). In contrast, the DNA-binding region of Chd1 is not observed in our structure and apparently mobile (Fig. 2b). We previously observed that the DNA-binding region binds the second DNA gyre in a nucleosome-Chd1 complex ${ }^{26}$ (Extended Data Fig. 5c). However, in the structure we present here, the second DNA gyre is no longer available for Chd1 interactions because DNA corresponding to SHL -7 to -5 has been transcribed by Pol II.

These observations explain how Chd1 is activated during transcription elongation (Extended Data Fig. 5c). As Pol II transcribes into the nucleosome, it displaces the DNA-binding region of Chd1 from DNA. The DNA-binding region is known to be an inhibitory domain that restricts Chd1 ATPase activity when engaged with nucleosomal DNA ${ }^{33}$. Displacement from DNA is predicted to release the inhibitory effect of the DNA-binding region ${ }^{33,34}$ and thereby activate Chd1. This results in DNA translocation toward the nucleosome dyad and into the Pol II cleft ${ }^{5}$, thereby facilitating Pol II progression.

Structure of Pol II-Spt4/5-nucleosome-FACT complex. To localize FACT during nucleosome transcription, we reconstituted a transcribing $S$. cerevisiae Pol II-Spt4/5-nucleosome-FACT complex by withholding Chd 1 from the assembly (Extended Data Fig. 2e-h). We employed single-particle cryo-EM to determine the structure of the complex at a nominal resolution of $3.1 \AA$ (Methods), with densities for FACT at lower local resolutions (Extended Data Fig. 6). The high-resolution density observed around the Pol II active site allowed us to unambiguously define the nucleic acid sequence register and revealed that Pol II had stalled with the active site located at bp 17 of the nucleosome (Extended Data Figs. 2g and 7). As in the first structure, Pol II adopts the post-translocated state (Extended Data Fig. 7d).

In this structure, four turns of nucleosomal DNA (SHL -7 to SHL -3 ) are unwrapped from the histone octamer, and the proximal H2A-H2B dimer is exposed (Fig. 3 and Supplementary Video 2). FACT embraces nucleosomal DNA near the dyad position at SHL +0.5 and contacts the template-strand DNA backbone with the middle domain of subunit Spt16 at SHL -0.5 (Fig. 3b,c). Compared to the structure of the Homo sapiens FACT-nucleosome complex ${ }^{27}$, the position of FACT appears shifted by one helical turn of DNA toward the side of the nucleosome that is distal to Pol II (Extended Data Fig. 8a). The previously observed position of FACT cannot be adopted in our structure because DNA is present on the distal side of the nucleosome but is absent in the isolated FACT-nucleosome complex structures that were reconstituted with shorter DNA to generate a subnucleosome without Pol II action ${ }^{27}$. In summary, our structure shows the position of FACT on a nucleosome that was partially unraveled by active Pol II transcription elongation.

Retention of the proximal $\mathrm{H} 2 \mathrm{~A}-\mathrm{H} 2 \mathrm{~B}$ dimer. In our second structure, FACT additionally binds the exposed proximal $\mathrm{H} 2 \mathrm{~A}-\mathrm{H} 2 \mathrm{~B}$ dimer using the C-terminal region of Spt16 (Fig. 3 and Extended Data Fig. 7h,i), as observed previously ${ }^{27,35}$. This finding is consistent with the known role of FACT in the retention of the proximal H2A$\mathrm{H} 2 \mathrm{~B}$ dimer during transcription ${ }^{4,36,37}$. Comparison with our first structure shows that the C-terminal region of Spt16 has replaced Spt5N for $\mathrm{H} 2 \mathrm{~A}-\mathrm{H} 2 \mathrm{~B}$ binding. This suggests that $\mathrm{Spt} 5 \mathrm{~N}$ retains the exposed proximal $\mathrm{H} 2 \mathrm{~A}-\mathrm{H} 2 \mathrm{~B}$ dimer until FACT is recruited. In our structure, FACT only binds the partially unwrapped nucleosome, and does not interact with Pol II or Spt4/5, consistent with recent results that showed recruitment of FACT to transcribed nucleosomes, rather than the transcribing complex, in vivo ${ }^{25,38}$.

Superposition of our two structures results in a clash between Chd1 and the FACT subunit Pob3 (Extended Data Fig. 8b). This indicates that binding of Chd1 and FACT to the nucleosome is mutually exclusive, at least in the highly defined states we trapped in our structures. Nevertheless, Chd1 binds FACT with its flexible N-terminal region (Extended Data Fig. 9), allowing one factor to remain loosely associated with the complex while the other factor binds the nucleosome directly. Additionally, binding of the C-terminal domain of Spt16 to the H2A-H2B dimer is predicted to prevent re-association of DNA with the exposed histone octamer surface, as had been observed in previous cryo-EM studies of Pol II-nucleosome complexes that showed unassigned DNA fragments of unknown origin ${ }^{6}$. Taken together, these observations support a dynamic mechanism with Chd1 and FACT acting either subsequently or in an alternating manner. 


\section{Discussion}

Based on our data and previously published data, we propose a model for how Chd1 and FACT mediate nucleosome transcription (Fig. 4 and Extended Data Fig. 10a). When the Pol II-Spt4/5 complex transcribes into a Chd1-bound nucleosome, it would release the DNA-binding region of Chd1. This activates the Chd1 translocase $\mathrm{e}^{33}$ and may facilitate Pol II progression. Pol II progression exposes the proximal $\mathrm{H} 2 \mathrm{~A}-\mathrm{H} 2 \mathrm{~B}$ dimer, which is temporarily stabilized by Spt5N binding. Further Pol II progression would then generate a binding site for FACT, which can then bind the partially unraveled nucleosome, leading to the displacement of Chd 1 and Spt5N. Modeling suggests a 30-bp window for FACT binding during Pol II progression (Extended Data Fig. 10b). Further Pol II progression would displace FACT from downstream DNA and enable FACT to transfer histones to upstream DNA or to relocate from the proximal to the distal $\mathrm{H} 2 \mathrm{~A}-\mathrm{H} 2 \mathrm{~B}$ dimer, thereby preventing loss of histones ${ }^{22,39}$.

It remains to be studied whether at each nucleosome both Chd 1 and FACT are essential for Pol II transcription. The order of events during nucleosome transcription also remain to be studied further, but it seems that Chd1 functions upstream of FACT because Chd1 can bind complete nucleosomes, whereas FACT can only bind partially unraveled nucleosomes. Chd1 may even recruit FACT because it is known to interact with $\mathrm{FACT}^{23,24,40}$. Indeed, we could confirm the Chd1-FACT interaction biochemically. This finding is consistent with the idea that FACT is recruited near the nucleosome by Chd 1 but remains flexible and only binds the nucleosome once DNA is partially unwrapped by transcription. In conclusion, we provide molecular snapshots of the dynamic process of factor-mediated nucleosome transcription and a model for Pol II progression through the nucleosome.

\section{Online content}

Any methods, additional references, Nature Research reporting summaries, source data, extended data, supplementary information, acknowledgements, peer review information; details of author contributions and competing interests; and statements of data and code availability are available at https://doi.org/10.1038/ s41594-021-00578-6.

Received: 21 January 2021; Accepted: 2 March 2021; Published online: 12 April 2021

\section{References}

1. Kornberg, R. D. Chromatin structure: a repeating unit of histones and DNA. Science 184, 868-871 (1974).

2. Luger, K., Mäder, A. W., Richmond, R. K., Sargent, D. F. \& Richmond, T. J. Crystal structure of the nucleosome core particle at $2.8 \AA$ resolution. Nature 389, 251 (1997).

3. Bondarenko, V. A. et al. Nucleosomes can form a polar barrier to transcript elongation by RNA polymerase II. Mol. Cell 24, 469-479 (2006).

4. Kireeva, M. L. et al. Nucleosome remodeling induced by RNA polymerase II loss of the H2A/H2B dimer during transcription. Mol. Cell 9, 541-552 (2002).

5. Farnung, L., Vos, S. M. \& Cramer, P. Structure of transcribing RNA polymerase II-nucleosome complex. Nat. Commun. 9, 5432 (2018).

6. Kujirai, T. et al. Structural basis of the nucleosome transition during RNA polymerase II passage. Science 362, 595-598 (2018)

7. Hodges, C., Bintu, L., Lubkowska, L., Kashlev, M. \& Bustamante, C. Nucleosomal fluctuations govern the transcription dynamics of RNA polymerase II. Science 325, 626-628 (2009)

8. Chen, Z. et al. High-resolution and high-accuracy topographic and transcriptional maps of the nucleosome barrier. Elife 8, e48281 (2019).

9. Teves, S. S., Weber, C. M. \& Henikoff, S. Transcribing through the nucleosome. Trends Biochem. Sci. 39, 577-586 (2014).

10. Petesch, S. J. \& Lis, J. T. Overcoming the nucleosome barrier during transcript elongation. Trends Genet. 28, 285-294 (2012)

11. Kulaeva, O. I., Hsieh, F.-K., Chang, H.-W., Luse, D. S. \& Studitsky, V. M. Mechanism of transcription through a nucleosome by RNA polymerase II. Biochim. Biophys. Acta 1829, 76-83 (2013).
12. Weber, C. M., Ramachandran, S. \& Henikoff, S. Nucleosomes are context-specific, H2A.Z-modulated barriers to RNA polymerase. Mol. Cell 53, 819-830 (2014).

13. Ehara, H. et al. Structural insight into nucleosome transcription by RNA polymerase II with elongation factors. Science 363, eaav8912 (2019).

14. Izban, M. G. \& Luse, D. S. Factor-stimulated RNA polymerase II transcribes at physiological elongation rates on naked DNA but very poorly on chromatin templates. J. Biol. Chem. 267, 13647-13655 (1992).

15. Kireeva, M. L. et al. Nature of the nucleosomal barrier to RNA polymerase II. Mol. Cell 18, 97-108 (2005).

16. Kim, J., Guermah, M. \& Roeder, R. G. The human PAF1 complex acts in chromatin transcription elongation both independently and cooperatively with SII/TFIIS. Cell 140, 491-503 (2010).

17. Smolle, M. et al. Chromatin remodelers Isw1 and Chd1 maintain chromatin structure during transcription by preventing histone exchange. Nat. Struct. Mol. Biol. 19, 884 (2012).

18. Skene, P. J., Hernandez, A. E., Groudine, M. \& Henikoff, S. The nucleosomal barrier to promoter escape by RNA polymerase II is overcome by the chromatin remodeler Chd1. Elife 3, e02042 (2014).

19. Lai, W. K. M. \& Pugh, B. F. Understanding nucleosome dynamics and their links to gene expression and DNA replication. Nat. Rev. Mol. Cell Biol. 18, 548-562 (2017).

20. Venkatesh, S. \& Workman, J. L. Histone exchange, chromatin structure and the regulation of transcription. Nat. Rev. Mol. Cell Biol. 16, 178-189 (2015).

21. Woodage, T., Basrai, M. A., Baxevanis, A. D., Hieter, P. \& Collins, F. S. Characterization of the CHD family of proteins. Proc. Natl Acad. Sci. USA 94, 11472-11477 (1997).

22. Orphanides, G., LeRoy, G., Chang, C.-H., Luse, D. S. \& Reinberg, D. FACT, a factor that facilitates transcript elongation through nucleosomes. Cell 92, 105-116 (1998).

23. Simic, R. et al. Chromatin remodeling protein Chd1 interacts with transcription elongation factors and localizes to transcribed genes. EMBO J. 22, 1846-1856 (2003).

24. Krogan, N. J. et al. RNA polymerase II elongation factors of Saccharomyces cerevisiae: a targeted proteomics approach. Mol. Cell. Biol. 22, 6979-6992 (2002).

25. Jeronimo, C. et al. FACT is recruited to the +1 nucleosome of transcribed genes and spreads in a Chd1-dependent manner. Preprint at bioRxiv https://doi.org/10.1101/2020.08.20.259960 (2020).

26. Farnung, L., Vos, S. M., Wigge, C. \& Cramer, P. Nucleosome-Chd1 structure and implications for chromatin remodelling. Nature 550, 539 (2017).

27. Liu, Y. et al. FACT caught in the act of manipulating the nucleosome. Nature 577, 426-431 (2020).

28. Cheung, A. C. M. \& Cramer, P. Structural basis of RNA polymerase II backtracking, arrest and reactivation. Nature 471, 249-253 (2011).

29. Meyer, P. A. et al. Structures and functions of the multiple KOW domains of transcription elongation factor Spt5. Mol. Cell. Biol. 35, 3354-3369 (2015).

30. Vasudevan, D., Chua, E. Y. D. \& Davey, C. A. Crystal structures of nucleosome core particles containing the '601' strong positioning sequence. J. Mol. Biol. 403, 1-10 (2010).

31. Bernecky, C., Plitzko, J. M. \& Cramer, P. Structure of a transcribing RNA polymerase II-DSIF complex reveals a multidentate DNA-RNA clamp. Nat. Struct. Mol. Biol. 24, 809-815 (2017).

32. Ehara, H. et al. Structure of the complete elongation complex of RNA polymerase II with basal factors. Science 357, 921-924 (2017).

33. Nodelman, I. M. et al. Interdomain communication of the Chd 1 chromatin remodeler across the DNA gyres of the nucleosome. Mol. Cell $\mathbf{6 5}$, 447-459.e6 (2017)

34. Nodelman, I. M. et al. The Chd1 chromatin remodeler can sense both entry and exit sides of the nucleosome. Nucleic Acids Res. 44, 7580-7591 (2016).

35. Mayanagi, K. et al. Structural visualization of key steps in nucleosome reorganization by human FACT. Sci. Rep. 9, 10183 (2019).

36. Belotserkovskaya, R. et al. FACT facilitates transcription-dependent nucleosome alteration. Science 301, 1090-1093 (2003).

37. Kulaeva, O. I. et al. Mechanism of chromatin remodeling and recovery during passage of RNA polymerase II. Nat. Struct. Mol. Biol. 16, 1272-1278 (2009).

38. Martin, B. J. E., Chruscicki, A. T. \& Howe, L. J. Transcription promotes the interaction of the facilitates chromatin transactions (FACT) complex with nucleosomes in Saccharomyces cerevisiae. Genetics 210, 869-881 (2018).

39. Hsieh, F.-K. et al. Histone chaperone FACT action during transcription through chromatin by RNA polymerase II. Proc. Natl Acad. Sci. USA 110, 7654-7659 (2013).

40. Kelley, D. E., Stokes, D. G. \& Perry, R. P. CHD1 interacts with SSRP1 and depends on both its chromodomain and its ATPase/helicase-like domain for proper association with chromatin. Chromosoma 108, 10-25 (1999).

Publisher's note Springer Nature remains neutral with regard to jurisdictional claims in published maps and institutional affiliations. 
(c) (i) Open Access This article is licensed under a Creative Commons

Attribution 4.0 International License, which permits use, sharing, adaptation, distribution and reproduction in any medium or format, as long as you give appropriate credit to the original author(s) and the source, provide a link to the Creative Commons license, and indicate if changes were made. The images or other third party material in this article are included in the article's Creative Commons license, unless indicated otherwise in a credit line to the material. If material is not included in the article's Creative Commons license and your intended use is not permitted by statutory regulation or exceeds the permitted use, you will need to obtain permission directly from the copyright holder. To view a copy of this license, visit http://creativecommons. org/licenses/by/4.0/.

(c) The Author(s) 2021 


\section{Methods}

No statistical methods were used to predetermine sample size. The experiments were not randomized, and the investigators were not blinded to allocation during experiments and outcome assessment

Molecular cloning. S. cerevisiae Spt 4 and Spt5 were cloned into vectors 438-A and 438-C, respectively, using ligation-independent cloning (LIC). Vectors 438-A and 438-C were a gift from S. Gradia (UC Berkeley), Addgene plasmids \#55218 and \#55220. Using LIC, the two genes were combined on a single 438-series vector. The construct contained Spt 5 with an $\mathrm{N}$-terminal $6 \times$ His tag followed by a maltose-binding protein tag, and a tobacco etch virus protease cleavage site. Spt4 did not contain tags. Each subunit in the combined vector was preceded by a PolH promoter and followed by a SV40 terminator. S. cerevisiae Spt6 was cloned into vector $438-\mathrm{C}$ using LIC. The construct contained an $\mathrm{N}$-terminal $6 \times$ His tag followed by a maltose-binding protein tag and a tobacco etch virus protease cleavage site. A codon-optimized sequence of $S$. cerevisiae TFIIS for expression in Escherichia coli was cloned into LIC-compatible vector 1-O. Vector 1-O was a gift from S. Gradia (UC Berkeley), Addgene plasmid \#29658. The construct contains an N-terminal $6 \times$ His tag followed by a Mocr solubility tag and a tobacco etch virus protease cleavage site.

Preparation of protein components. S. cerevisiae Pol II was purified as described previously $^{41}$. S. cerevisiae Chd 1 and FACT were expressed and purified as described $^{26}$. All insect cell lines used for expression were purchased from Life Technologies (Sf9, Sf21) or from Expression Systems (Hi5) and used as identified by the vendor. Cell lines were not tested for mycoplasma contamination.

S. cerevisiae Spt 4 and Spt5 were co-expressed in insect cells using a similar approach as that reported previously ${ }^{42}$. After harvest, cell pellets were resuspended in lysis buffer $500(500 \mathrm{mM} \mathrm{NaCl}, 20 \mathrm{mM} \mathrm{Na}$-HEPES, pH 7.4, $10 \%$ (v/v) glycerol, $1 \mathrm{mM}$ DTT, $30 \mathrm{mM}$ imidazole, $\mathrm{pH} 8.0,0.284 \mu \mathrm{g} \mathrm{ml}^{-1}$ leupeptin, $1.37 \mu \mathrm{g} \mathrm{ml}^{-1}$ pepstatin A, $0.17 \mathrm{mg} \mathrm{ml}^{-1}$ PMSF, $0.33 \mathrm{mg} \mathrm{ml}^{-1}$ benzamidine). Cells were lysed by sonication. The cell lysate was subjected to centrifugation $\left(18,000 \mathrm{~g}, 4^{\circ} \mathrm{C}, 30 \mathrm{~min}\right)$ and ultracentrifugation $\left(235,000 \mathrm{~g}, 4^{\circ} \mathrm{C}, 60 \mathrm{~min}\right)$. The supernatant containing Spt $4 / 5$ was subsequently filtered using $0.2-\mu \mathrm{m}$ syringe filters (Millipore). The filtered supernatant was applied to a GE HisTrap $5 \mathrm{ml} \mathrm{HP} \mathrm{(GE} \mathrm{Healthcare),}$ pre-equilibrated in lysis buffer. The column was subsequently washed with three column volumes (CV) lysis buffer 500, $3 \mathrm{CV}$ high-salt buffer $(1000 \mathrm{mM} \mathrm{NaCl}$, $20 \mathrm{mM}$ Na-HEPES, pH 7.4, 10\% (v/v) glycerol, $1 \mathrm{mM}$ DTT, $30 \mathrm{mM}$ imidazole, $\mathrm{pH} 8.0,0.284 \mu \mathrm{g} \mathrm{ml}^{-1}$ leupeptin, $1.37 \mu \mathrm{g} \mathrm{ml}^{-1}$ pepstatin $\mathrm{A}, 0.17 \mathrm{mg} \mathrm{ml}^{-1} \mathrm{PMSF}$, $0.33 \mathrm{mg} \mathrm{ml}^{-1}$ benzamidine) and $4.5 \mathrm{CV}$ lysis buffer. Bound protein was eluted by gradient over $9 \mathrm{CV}$ to $100 \%$ nickel elution buffer $(500 \mathrm{mM} \mathrm{NaCl}, 20 \mathrm{mM}$ Na-HEPES, pH 7.4, $10 \%$ (v/v) glycerol, 1 mM DTT, $500 \mathrm{mM}$ imidazole, $\mathrm{pH} 8.0$ $0.284 \mu \mathrm{g} \mathrm{ml}^{-1}$ leupeptin, $1.37 \mu \mathrm{g} \mathrm{ml}^{-1}$ pepstatin A, $0.17 \mathrm{mg} \mathrm{ml}^{-1}$ PMSF, $0.33 \mathrm{mg} \mathrm{ml}^{-1}$ benzamidine) over $9 \mathrm{CV}$. Fractions containing Spt $4 / 5$ were pooled and dialyzed for $16 \mathrm{~h}$ against dialysis buffer $(300 \mathrm{mM} \mathrm{NaCl}, 20 \mathrm{mM} \mathrm{Na}$-HEPES, $\mathrm{pH} 7.4,10 \%$ (v/v) glycerol, $1 \mathrm{mM} \mathrm{DTT,} 30 \mathrm{mM}$ imidazole, $\mathrm{pH}$ 8.0, $0.284 \mu \mathrm{g} \mathrm{ml}^{-1}$ leupeptin, $1.37 \mu \mathrm{g} \mathrm{ml}^{-1}$ pepstatin A, $0.17 \mathrm{mg} \mathrm{ml}^{-1}$ PMSF, $0.33 \mathrm{mg} \mathrm{ml}^{-1}$ benzamidine). The dialyzed sample was applied to a GE HisTrap $5 \mathrm{ml} \mathrm{HP}$ and GE HiTrap Q $5 \mathrm{ml} \mathrm{HP}$ column combination. After application of the sample, the HisTrap $5 \mathrm{ml}$ HP was removed, and the HiTrap Q $5 \mathrm{ml} \mathrm{HP}$ was washed with $5 \mathrm{CV}$ dialysis buffer. Spt4/5 was eluted using a gradient elution to $100 \%$ high-salt buffer over 9 CV. Fractions containing Spt4/5 were concentrated using an Amicon Millipore $15 \mathrm{ml}$ 50,000 MWCO centrifugal concentrator and applied to a GE Superdex 200 Increase $10 / 300 \mathrm{GL}$ size exclusion column, pre-equilibrated in gel filtration buffer $(500 \mathrm{mM}$ $\mathrm{NaCl}, 20 \mathrm{mM}$ Na-HEPES, pH 7.4, 10\% (v/v) glycerol, 1 mM DTT). Peak fractions were analyzed with SDS-PAGE. Fractions containing Spt $4 / 5$ were concentrated using an Amicon Millipore $15 \mathrm{ml}$ 50,000 MWCO centrifugal concentrator to a concentration of $\sim 20 \mu \mathrm{M}$, aliquoted, flash frozen and stored at $-80^{\circ} \mathrm{C}$. Typical preparations yielded $300 \mu \mathrm{g}$ of Spt $4 / 5$ from $1.2 \mathrm{~L}$ of insect cell culture.

S. cerevisiae Spt6 was expressed in insect cells and subsequently purified with a similar protocol used for $H$. sapiens SPT6 (ref. ${ }^{43}$ ) with a final concentration of $\sim 60 \mu \mathrm{M}$. Typical yields are $\sim 10 \mathrm{mg}$ from $1.2 \mathrm{~L}$ of insect cell culture.

S. cerevisiae TFIIS was expressed in E. coli BL21 (DE3) RIL cells grown in $\mathrm{LB}$ medium. Cells were grown to an optical density at $600 \mathrm{~nm}$ of 0.6 at $37^{\circ} \mathrm{C}$. Expression of TFIIS was subsequently induced with $1 \mathrm{mM}$ isopropyl $\beta$-D1-thiogalactopyranoside at $18^{\circ} \mathrm{C}$ for $16 \mathrm{~h}$. Cells were harvested by centrifugation and resuspended in TFIIS lysis buffer $(300 \mathrm{mM} \mathrm{NaCl}, 20 \mathrm{mM}$ Na-HEPES, $\mathrm{pH}$ 7.4, $10 \%$ (v/v) glycerol, $1 \mathrm{mM}$ DTT, $30 \mathrm{mM}$ imidazole, $\mathrm{pH} 8.0,0.284 \mu \mathrm{g} \mathrm{ml}^{-1}$ leupeptin, $1.37 \mu \mathrm{g} \mathrm{ml}^{-1}$ pepstatin A, $0.17 \mathrm{mg} \mathrm{ml}^{-1}$ PMSF, $0.33 \mathrm{mg} \mathrm{ml}^{-1}$ benzamidine). Cells were lysed by sonication. Two rounds of centrifugation $\left(87,000 \mathrm{~g}, 4^{\circ} \mathrm{C}, 30 \mathrm{~min}\right)$ were used to clear the lysate. The supernatant was applied to a GE HisTrap $5 \mathrm{ml}$ HP. Affinity purification, dialysis and TEV digest were performed similarly as described for Spt4/5. The dialyzed and TEV-digested sample was applied to a GE HisTrap $5 \mathrm{ml} \mathrm{HP}$, pre-equilibrated in TFIIS lysis buffer. The flow-through containing TFIIS was collected and concentrated using an Amicon Millipore $15 \mathrm{ml}$ 10,000 MWCO centrifugal concentrator and applied to a GE S75 16/600 pg size exclusion column, pre-equilibrated in TFIIS size exclusion buffer $(300 \mathrm{mM} \mathrm{NaCl}$, $20 \mathrm{mM}$ Na-HEPES, pH 7.4, 10\% (v/v) glycerol, $1 \mathrm{mM} \mathrm{DTT}$ ). Fractions containing TFIIS were concentrated to $\sim 300 \mu \mathrm{M}$, aliquoted, flash frozen and stored at $-80^{\circ} \mathrm{C}$.
Typical yields were $10 \mathrm{mg}$ from $6 \mathrm{~L}$ of $E$. coli expression culture. Protein identity of all protein components was confirmed by MS.

Nucleosome preparation. Xenopus laevis histones were expressed and purified as described previously ${ }^{26,44}$. Histone $\mathrm{H} 3$ was $\mathrm{H} 3 \mathrm{~K} 36 \mathrm{Cme} 3$ modified $^{45}$. DNA fragments for nucleosome reconstitution were generated by PCR essentially as described previously ${ }^{27,36}$. A vector containing a modified Widom 601 sequence was used as a template for PCR. Super-helical locations were assigned based on previous publications $s^{5,6,26,46}$, assuming direction of transcription from negative to positive SHLs. Large-scale PCR reactions were performed with two PCR primers (forward primer: 5'-ACG AAG CGT AGC ATC ACT GTC TTG-3'; reverse primer: 5'-ATC AGA ATC CCG GTG CCG AGG CCG C-3') at a scale of $50 \mathrm{ml}$. Full-length PCR product is reported in Supplementary Data. PCR products were purified using anion exchange chromatography (GE Resoure Q $6 \mathrm{ml}$ ) followed by ethanol precipitation. The DNA product was digested with TspRI in 1X CutSmart Buffer (NEB) overnight at $65^{\circ} \mathrm{C}$ to generate the 9 -nt single-stranded DNA overhang. The digestion product was again purified with anion exchange chromatography, ethanol precipitated and resuspended in water. Nucleosome core particle reconstitution was then performed using the salt-gradient dialysis method ${ }^{44}$. The resulting nucleosome was purified using a Model 491 Prep Cell (Bio-Rad) and subsequently concentrated to 10-20 $\mathrm{MM}$ using an Amicon Millipore $15 \mathrm{ml}$ 50,000 MWCO centrifugal concentrator. Quantification of the purified nucleosome was achieved by measuring absorbance at $280 \mathrm{~nm}$. Molar extinction coefficients at $280 \mathrm{~nm}$ were determined for protein and nucleic acid components and were summed to yield a molar extinction coefficient for the reconstituted extended nucleosome.

RNA extension assays. RNA extension assays were performed on the same nucleosomal template substrate used for the structural studies. A 6-FAM 5'-labelled 11-nt RNA (5'-/56-FAM/ rUrUrA rUrCrA rCrUrG rUrC-3') was employed to monitor the transcription reaction. TFIIS has been added to all transcription reactions to prevent formation of overextended DNA-RNA hybrids and facilitate nucleosome passage $\mathrm{e}^{5,6}$. The position of Pol II pausing was assigned by indicating the position of the Pol II active site on the Widom 601 DNA. This provided an unambiguous assignment at nucleotide resolution. Therefore, our pausing sites at bp 1 and bp 42 correspond to the previously described ${ }^{6,13}$ pause sites, with the Pol II leading edge at SHL -5 and SHL -1 , respectively.

All subsequent concentrations refer to the concentration in the final reaction. The final concentrations of buffer components were $130 \mathrm{mM} \mathrm{NaCl}, 20 \mathrm{mM}$ Na-HEPES, pH 7.4, $3 \mathrm{mM} \mathrm{MgCl}_{2}, 4 \%$ (v/v) glycerol, $1 \mathrm{mM}$ DTT/TCEP. The final volume for each RNA extension reaction was $40 \mu \mathrm{l}$. RNA $(80 \mathrm{nM})$, nucleosomal template $(80 \mathrm{nM})$ and $S$. cerevisiae Pol II $(100 \mathrm{nM})$ were mixed in equimolar ratios and incubated for $5 \mathrm{~min}$ on ice. Spt4/5 (120 nM) and additional factors (500 nM each), $10 \times$ compensation buffer and water were added to achieve final assay conditions. The sample was incubated for $3 \mathrm{~min}$ at $30^{\circ} \mathrm{C}$. Transcription elongation was started by the addition of ATP, CTP, GTP and UTP ( $1 \mathrm{mM}$ each) and TFIIS $(60 \mathrm{nM})$. Five microliters of the reactions were quenched after $5 \mathrm{~min}, 10 \mathrm{~min}$ and $30 \mathrm{~min}$ in $5 \mu \mathrm{l} 2 \times$ stop buffer (6.4 M urea, $50 \mathrm{mM}$ EDTA, pH 8.0, $1 \times$ TBE buffer) if time courses were performed. Samples were treated with $4 \mu \mathrm{g}$ proteinase $\mathrm{K}$ for $15 \mathrm{~min}$ at $37^{\circ} \mathrm{C}$, denatured at $95^{\circ} \mathrm{C}$ for $3 \mathrm{~min}$, and separated by denaturing gel electrophoresis $(4 \mu \mathrm{l}$ of sample applied to an $8 \mathrm{M}$ urea, $1 \times$ TBE buffer, $12 \%$ acrylamide:bis-acrylamide 19:1 gel, run in $0.5 \times$ TBE buffer at $300 \mathrm{~V}$ for $30 \mathrm{~min}$ ). RNA extension products were visualized using the 6-FAM label and a Typhoon 9500 FLA imager at an excitation wavelength of $473 \mathrm{~nm}$ and emission wavelength range of $>520 \mathrm{~nm}$.

Gels were subjected to linear contrast enhancement. Source data for all quantified RNA extension assays are provided in Source Data Fig. 1. All RNA extension assays were performed independently and at least three times. Full-length RNA extension products were quantified using Fiji 1.0. The products were normalized against the total intensity of the respective reaction lane to control for errors during gel loading. Bar charts show mean values and standard deviation as error bars. The following $P$ values were applied ${ }^{\star} P<0.05$, ${ }^{*} P<0.01$. A two-tailed $t$ test was employed to determine statistical significance.

Reconstitution of transcribing Pol II-nucleosome complexes. Complexes for cryo-EM were formed in a final buffer containing $130 \mathrm{mM} \mathrm{NaCl}, 20 \mathrm{mM}$ Na-HEPES, pH 7.4, 3 mM MgCl 2,1 mM DTT/TCEP, 4\% (v/v) glycerol. RNA (480 pmol, same construct as used for RNA extension assays) and nucleosome $(120 \mathrm{pmol})$ were incubated for $5 \mathrm{~min}$ on ice. Pol II $(120 \mathrm{pmol}), \mathrm{Spt} 4 / 5(180 \mathrm{pmol})$ and Spt6 (180 pmol) were added and incubated for $5 \mathrm{~min}$ on ice. Water and compensation buffer were added to reach final buffer conditions, and the sample was incubated for $5 \mathrm{~min}$. Transcription elongation was started by the addition of $1 \mathrm{mM}$ each of GTP, CTP and UTP and $0.4 \mathrm{mM} 3^{\prime}$-dATP in the case of the Pol II-Spt4/5-nucleosome-FACT complex. Instead of $3^{\prime}$-dATP, $1 \mathrm{mM} \mathrm{ADP-BeF}$ was added to the Pol II-Spt4/5-nucleosome-Chd1-FACT complex. TFIIS (108 pmol) was immediately added after addition of NTP.

After $15 \mathrm{~min}$ of incubation at $30^{\circ} \mathrm{C}$, Chd $1(180 \mathrm{pmol})$ and FACT $(180 \mathrm{pmol})$, preincubated with $\mathrm{H} 2 \mathrm{~A}-\mathrm{H} 2 \mathrm{~B}$ dimer $(180 \mathrm{pmol})$, or FACT alone (180 pmol), preincubated with $\mathrm{H} 2 \mathrm{~A}-\mathrm{H} 2 \mathrm{~B}$ dimer $(180 \mathrm{pmol})$, were added. The transcription reactions were allowed to proceed for an additional $30 \mathrm{~min}$ at $30^{\circ} \mathrm{C}$ and quenched 
with EDTA ( $10 \mathrm{mM}$ final concentration). The samples were subsequently centrifuged and applied onto a Superose $63.2 / 300$ Increase size exclusion column (GE Healthcare), equilibrated in complex buffer ( $100 \mathrm{mM} \mathrm{NaCl}, 20 \mathrm{mM}$ $\mathrm{Na}-\mathrm{HEPES}, \mathrm{pH}$ 7.4, $3 \mathrm{mM} \mathrm{MgCl}_{2}, 1 \mathrm{mM}$ TCEP, 4 \% (v/v) glycerol). Fractions eluted from the size exclusion column were analyzed using SDS-PAGE and denaturing-urea PAGE ( $8 \mathrm{M}$ urea, $1 \times$ TBE buffer, $12 \%$ acrylamide:bis-acrylamide 19:1 gel). Consistent with previous observations ${ }^{5}$, TFIIS is lost from the elongation complex during size exclusion chromatography. Fractions containing the complex were individually crosslinked and dialyzed against dialysis buffer (100 mM NaCl, $20 \mathrm{mM} \mathrm{Na-HEPES,} \mathrm{pH} \mathrm{7.4,} 3 \mathrm{mM} \mathrm{MgCl}_{2}, 1 \mathrm{mM}$ TCEP) for $3 \mathrm{~h}$, as described previously ${ }^{4}$.

The dialyzed complexes with an approximate concentration of $50-100 \mathrm{nM}$ were applied to R2/2 gold grids, Au 200 mesh (Quantifoil). The grids were glow discharged for $100 \mathrm{~s}$ prior to application of $2 \mu \mathrm{l}$ of sample to each side of the grid. After incubation of the sample for $8 \mathrm{~s}$, the grid was blotted and vitrified by plunging into liquid ethane using a Vitrobot Mark IV (Thermo Fisher). The Vitrobot was operated at $4{ }^{\circ} \mathrm{C}$ and $100 \%$ humidity. A blot force of 5 and blot time between 3 and $5 \mathrm{~s}$ were used for the sample preparation. The grids were clipped and subsequently stored in liquid nitrogen before data acquisition.

Cryo-EM analysis and image processing. Cryo-EM data were collected on a Titan Krios II transmission electron microscope (FEI) operated at $300 \mathrm{keV}$. A K3 summit direct detector (GATAN) with a GIF Quantum Filter with a slit width of $20 \mathrm{eV}$ was used for the data acquisition. Data acquisition was performed using SerialEM at a nominal magnification of $81,000 \times$, corresponding to a pixel size of $1.05 \AA$ per pixel in nanoprobe EF-TEM mode. For the Pol II-Spt4/5-nucleosome-Chd1 dataset, image stacks of 40 frames were collected in counting mode over $2.2 \mathrm{~s}$. The dose rate was $\sim 18.12 \mathrm{e}^{-}$per $\AA^{2}$ per s for a total dose of $39.87 \mathrm{e}^{-}$per $\AA^{2}$. For the Pol II-Spt4/5-nucleosome-FACT dataset, image stacks of 40 frames were collected in counting mode over $2.2 \mathrm{~s}$. The dose rate was $\sim 18.30 \mathrm{e}-$ per $\AA^{2}$ per s for a total dose of $40.25 \mathrm{e}^{-}$per $\AA^{2}$

Micrographs were stacked and processed using Warp ${ }^{48}$. CTF estimation and motion correction was performed using Warp ${ }^{48}$. Particles were picked using an in-house trained instance of the neural network BoxNet 2 as implemented in Warp, yielding 3,755,390 particles for the Pol II-Spt4/5-nucleosome-Chd1 dataset and $5,227,093$ particles for the Pol II-Spt4/5-nucleosome-FACT dataset. Particles were extracted with a box size of 400 pixels and normalized. Further image processing was performed with cryoSPARC ${ }^{49}$ and RELION 3.0.7.

For the Pol II-Spt4/5-nucleosome-Chd1 dataset, particles were separated into two subsets and subsequently $3 \mathrm{D}$ classified using cryoSPARC ${ }^{49}$. Particles were selected for the presence of a nucleosome-like density. Selected particles were imported into RELION ${ }^{50}$. Two subsequent rounds of 3D classification resulted in 247,604 particles with clear nucleosomal density. A 3D refinement of these particles resulted in an overall model of $2.6 \AA$. The particles were CTF refined, and Bayesian polishing was conducted. A mask encompassing the nucleosome and additional density at SHL +2 was applied to two rounds of 3D classification to select for particles that contain Chd1. This resulted in 30,876 particles with clear density for Chd1 bound to the partially unwrapped nucleosome. Particles were then subsequently $3 \mathrm{D}$ refined resulting in map A (EMD-12666) with a resolution of $2.9 \AA$ (FSC threshold 0.143 criterion). The map showed excellent density for Pol II, but the nucleosome-Chd1 part of the map showed flexibility. Therefore, Pol II with Spt $4 / 5$ and the nucleosome-Chd 1 parts of the maps were individually refined using a mask for Pol II-Spt $4 / 5$ or nucleosome-Chd1, respectively. Signal subtraction was applied. This resulted in two masked refinements (Pol II-Spt4/5, map B, EMD-12667; nucleosome-Chd1 with signal subtraction, map C,

EMD-12668) with overall improved density. These two masked refinements were combined using the Frankenmap and Noise2map tool set of Warp, resulting in the final composite map (map D, EMD-12449).

For the Pol II-Spt4/5-nucleosome-FACT dataset, particles were separated into two subsets and subsequently 3D classified using cryoSPARC and RELION. Particles were selected for the presence of nucleosome-like densities. The selection resulted in 603,550 particles with clear nucleosome-like density. The selected particles were $3 \mathrm{D}$ refined using an angular sampling rate of $7.5^{\circ}$ and subjected to further classifications to select for particles with bound FACT. This ultimately resulted in 48,718 particles. These particles were again subjected to 3D classification. After 3D refinement, CTF refinement and Bayesian polishing, the remaining 47,138 particles resulted in a final refinement (map 1, EMD-12669) with an overall resolution of $3.1 \AA$ (FSC threshold 0.143 criterion). To improve densities for the Pol II-Spt $4 / 5$ and nucleosome-FACT parts of the map, the particles were subjected to masked refinements (maps 2, EMD-12670 and map 3, EMD-12671). Similar to the Chd1 dataset, the masked refinements were combined using the Frankenmap and Noise2map tools included in Warp, yielding the final map (composite map 4, EMD-12450). Local resolutions of the composite maps were estimated using the RELION built-in tool for the determination of local resolutions.

Model building and refinement. For the Pol II-Spt4/5-nucleosome-Chd 1 structure, structures of $S$. cerevisiae $\mathrm{Pol}$ II (PDB 3PO2), X. laevis nucleosome (PDB 3LZ0), Chd1 with ADP-BeF 3 (PDB 5O9G) and Spt4/5 (PDB 2EXU) were rigid-body docked into map D and refined using $\mathrm{Coot}^{51}$. DNA from the elongation complex and nucleosomal DNA were connected using Coot. Density in the active site of Pol II allowed unambiguous assignment of DNA register. Surprisingly, the complex had transcribed over the T-less cassette that should stall further

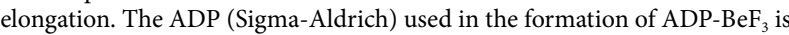
reported to be contaminated with up to $2.76 \% \mathrm{ATP}^{52}$, possibly providing the required substrate to transcribe past the end of the T-less cassette. Identification of DNA-RNA register was aided by map $B$.

For the Pol II-Spt4/5-nucleosome-FACT structure, the refined Pol II part of the Pol II-Spt4/5-nucleosome-Chd1 structure was rigid-body docked into the density. Additionally, X. laevis nucleosome (PDB 3LZ0), H. sapiens FACT (PDB $6 \mathrm{UPK}$ ), and Spt4/5 (PDB 2EXU) were rigid-body docked into map 4 using Coot. S. cerevisiae FACT structures (Spt16, PDB 4IOY; Pob3, PDB 4PQ0) were then superposed onto the docked $H$. sapiens FACT structure. The dimerization domain of Spt16 and Pob3 were generated using PHYRE2 (ref. ${ }^{53}$ ) and superposed onto the docked FACT structure without any additional manual manipulation. The CTD of Spt16 was modeled de novo as a polyalanine extension of Spt16 with no sequence assignment. DNA from the Pol II part of the structure and the nucleosomal DNA were connected in Coot. The density in the active site allowed for unambiguous assignment of the DNA register, and the nucleic acid sequence was adjusted accordingly. Identification of DNA-RNA register was aided by map 2 .

Both atomic models were real-space refined using PHENIX ${ }^{54}$, with secondary structure restraints against map D (Pol II-Spt4/5-nucleosome-Chd1 model) and map 4 (Pol II-Spt4/5-nucleosome-FACT model).

Figure generation. Figures for structural models were generated using PyMol (version 2.3.4; https://pymol.org/), UCSF Chimera ${ }^{55}$ and UCSF ChimeraX ${ }^{56}$. Gel quantification was performed using Fiji, and graphs were generated using GraphPad Prism.

Reporting Summary. Further information on research design is available in the Nature Research Reporting Summary linked to this article.

\section{Data availability}

The cryo-EM reconstructions and final models for the Pol II-Spt4/5-nucleosomeChd1 complex were deposited with the Electron Microscopy Data Base (EMD-12449) and the Protein Data Bank (PDB 7NKX). The cryo-EM reconstructions and final models for the Pol II-Spt4/5-nucleosome-FACT complex were deposited with the Electron Microscopy Data Base (EMD-12450) and with the Protein Data Bank (PDB 7NKY). For the Pol II-Spt4/5-nucleosome-Chd1 complex, maps A-C were deposited as EMD-12666, EMD-12667 and EMD-12668, respectively. For the Pol II-Spt4/5-nucleosome-FACT complex, maps 1-3 were deposited as EMD-12669, EMD-12670 and EMD-12671, respectively. Source data are provided with this paper.

\section{References}

41. Sydow, J. F. et al. Structural basis of transcription: mismatch-specific fidelity mechanisms and paused RNA polymerase II with frayed RNA. Mol. Cell 34, 710-721 (2009)

42. Vos, S. M. et al. Architecture and RNA binding of the human negative elongation factor. Elife 5, e14981 (2016).

43. Vos, S. M. et al. Structure of activated transcription complex Pol II-DSIFPAF-SPT6. Nature 560, 607-612 (2018).

44. Dyer, P. N. et al. Reconstitution of nucleosome core particles from recombinant histones and DNA. Methods Enzymol. 375, 23-44 (2004).

45. Simon, M. D. \& Shokat, K. M. in Methods in Enzymolology Vol. 512 (eds. Wu, C. \& Allis, C. D.) Ch. 3 (Elsevier, 2012).

46. Sundaramoorthy, R. et al. Structure of the chromatin remodelling enzyme Chd1 bound to a ubiquitinylated nucleosome. Elife 7, e35720 (2018).

47. Vos, S. M., Farnung, L., Linden, A., Urlaub, H. \& Cramer, P. Structure of complete Pol II-DSIF-PAF-SPT6 transcription complex reveals RTF1 allosteric activation. Nat. Struct. Mol. Biol. 27, 668-677 (2020).

48. Tegunov, D. \& Cramer, P. Real-time cryo-electron microscopy data preprocessing with Warp. Nat. Methods 16, 1146-1152 (2019).

49. Punjani, A., Rubinstein, J. L., Fleet, D. J. \& Brubaker, M. A. cryoSPARC: algorithms for rapid unsupervised cryo-EM structure determination. Nat. Methods 14, 290-296 (2017).

50. Zivanov, J. et al. New tools for automated high-resolution cryo-EM structure determination in RELION-3. Elife 7, e42166 (2018).

51. Emsley, P., Lohkamp, B., Scott, W. G. \& Cowtan, K. Features and development of Coot. Acta Crystallogr. D Biol. Crystallogr. 66, 486-501 (2010)

52. Winardhi, R. S., Tang, Q., You, H., Sheetz, M. \& Yan, J. The holdase function of Escherichia coli Hsp70 (DnaK) chaperone. Preprint at bioRxiv https://doi. org/10.1101/305854 (2018).

53. Kelley, L. A., Mezulis, S., Yates, C. M., Wass, M. N. \& Sternberg, M. J. E. The Phyre 2 web portal for protein modeling, prediction and analysis. Nat. Protoc. 10, 845-858 (2015). 
54. Afonine, P. V. et al. Real-space refinement in PHENIX for cryo-EM and crystallography. Acta Crystallogr. Sect. D Struct. Biol. 74, 531-544 (2018).

55. Pettersen, E. F. et al. UCSF Chimera-a visualization system for exploratory research and analysis. J. Comput. Chem. 25, 1605-1612 (2004).

56. Goddard, T. D. et al. UCSF ChimeraX: meeting modern challenges in visualization and analysis. Protein Sci. 27, 14-25 (2018)

\section{Acknowledgements}

We thank C. Oberthür and F. Grabbe for help with protein purification, U. Neef and P. Rus for insect cell maintenance, and C. Dienemann and U. Steuerwald for maintenance of cryo-EM resources. We thank S. M. Vos for critical reading and input. M.E. is funded by the Deutsche Forschungsgemeinschaft (DFG, German Research Foundation) under Germany's Excellence Strategy - EXC 2067/1- 390729940. P.C. was supported by the Deutsche Forschungsgemeinschaft (SFB860, SPP2191, EXC 2067/1- 390729940) and the ERC Advanced Investigator Grant CHROMATRANS (grant agreement no. 693023).

\section{Author contributions}

L.F. designed and conducted experiments and interpreted data, unless stated otherwise. M.O. assisted with the purification of Spt4/5 and RNA extension assays.
L.F. and M.O. prepared the Pol II-Spt4/5-nucleosome-Chd1 and Pol II-Spt4/5nucleosome-FACT complexes for cryo-EM. M.E. conducted initial FACT-nucleosome binding experiments. P.C. supervised research. L.F. and P.C. wrote the manuscript with input from all authors.

\section{Competing interests}

The authors declare no competing interests.

\section{Additional information}

Extended data is available for this paper at https://doi.org/10.1038/s41594-021-00578-6.

Supplementary information The online version contains supplementary material available at https://doi.org/10.1038/s41594-021-00578-6.

Correspondence and requests for materials should be addressed to P.C.

Peer review information Beth Moorefield was the primary editor on this article and managed its editorial process and peer review in collaboration with the rest of the editorial team.

Reprints and permissions information is available at www.nature.com/reprints. 


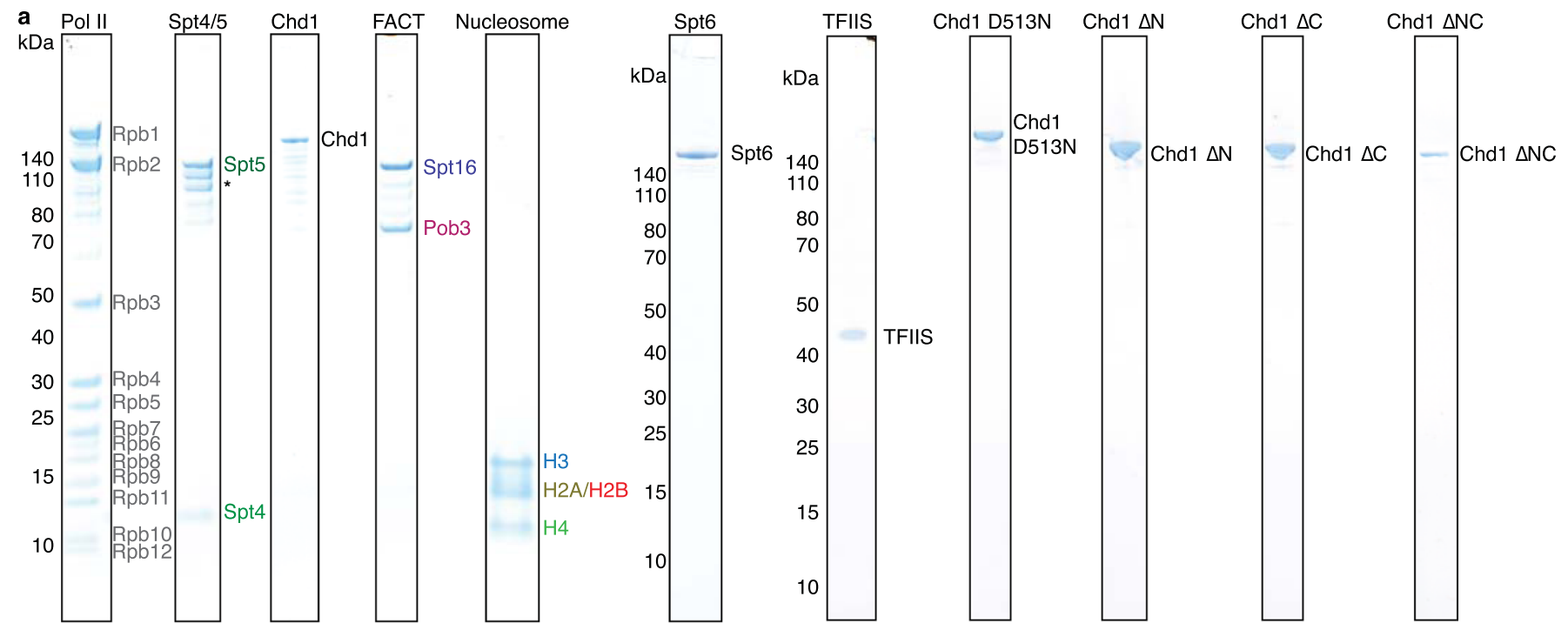

b

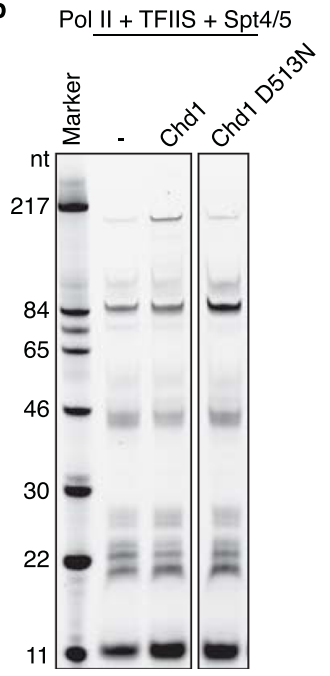

C

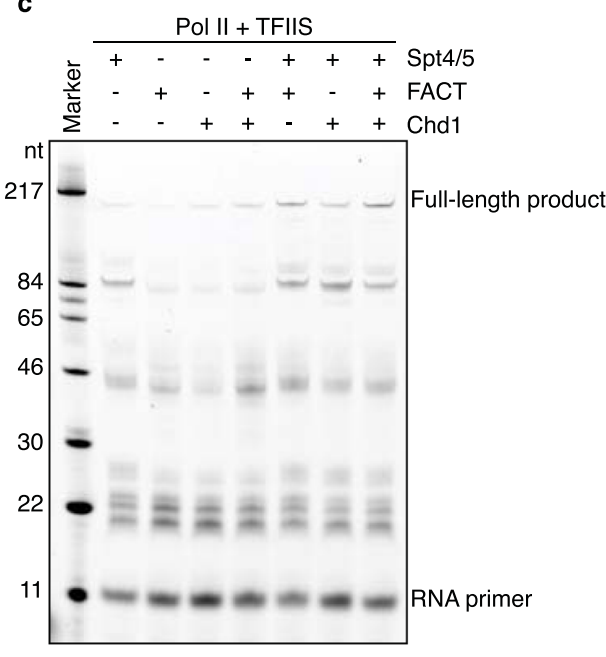

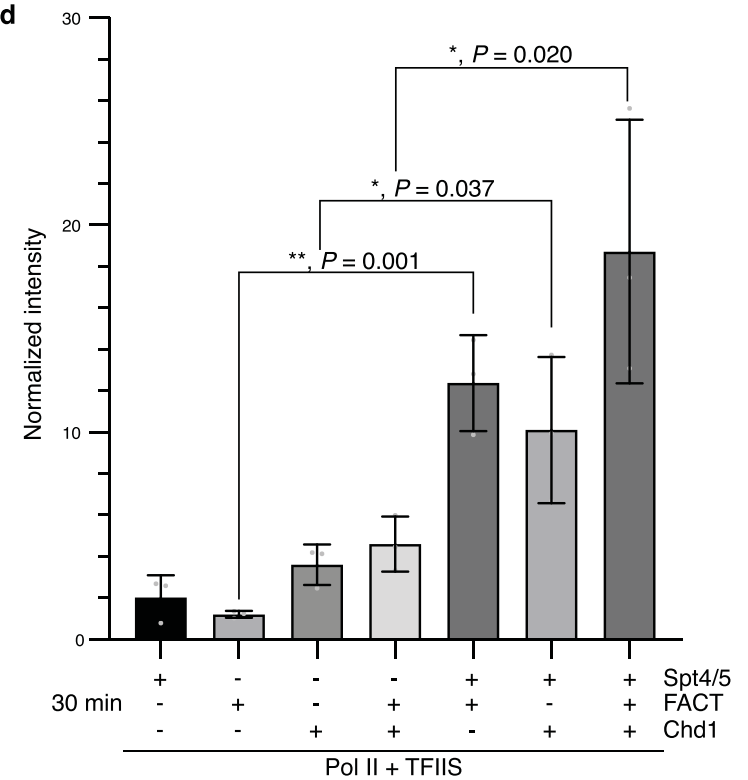

Extended Data Fig. 1 | Additional information on RNA extension assays. a, SDS-PAGE of purified proteins. Purified proteins were run on 4-12 \% Bis-Tris SDS-PAGE gels in 1X MES Buffer, stained with Coomassie Blue. Asterisk $\left(^{\star}\right)$ demarcates degradation products of Spt5. b, $12 \%$ denaturing urea gel of RNA extension assay with Chd1 D513N mutant. RNA is visualized using 6-FAM label on $5^{\prime}$ end of RNA. c, $12 \%$ denaturing urea gel of RNA extension assay with different factor combinations reveals dependence of Spt4/5 for increase in full-length product in the presence of FACT and Chd1. RNA is visualized using 6-FAM label on 5' end of RNA. d, Bar plot with quantification of c. Mean normalized intensity is shown as bar plot. Error bars represent standard deviation. Quantification from $n=3$ independent experiments with ${ }^{\star} P<0.05$, ${ }^{\star \star} P<0.01$ with two-tailed t-test. Uncropped gel images in $\mathbf{a}, \mathbf{b}, \mathbf{c}$ and data for the plots in $\mathbf{d}$ are available as Source Data Extended Data Fig. 1 and Supplementary Data. 


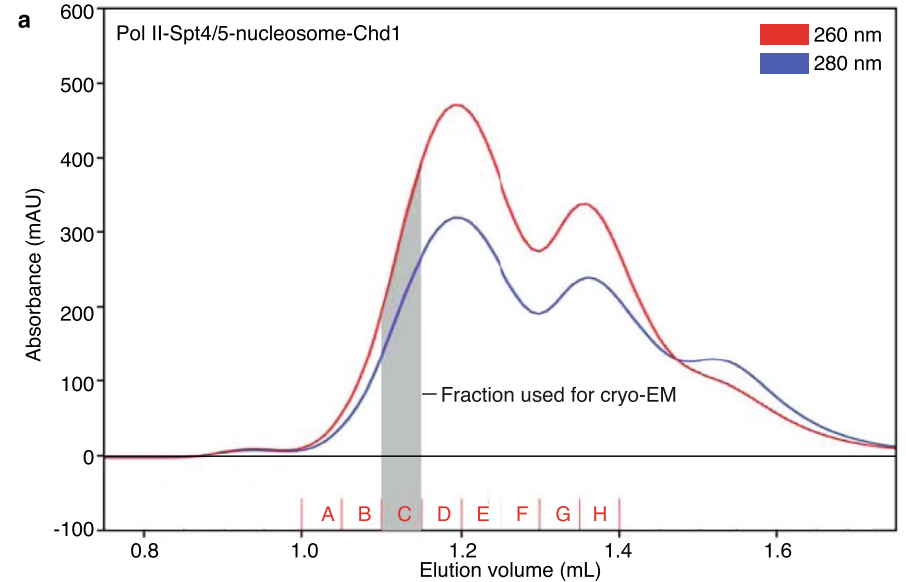

c

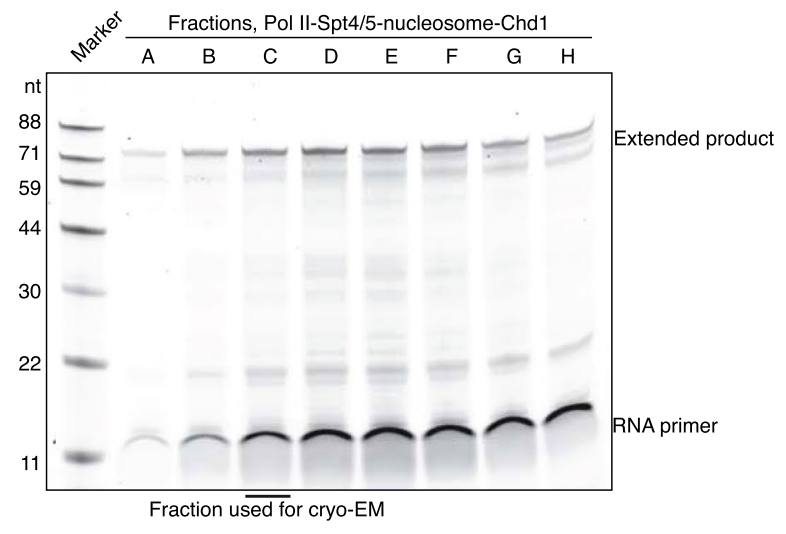

e

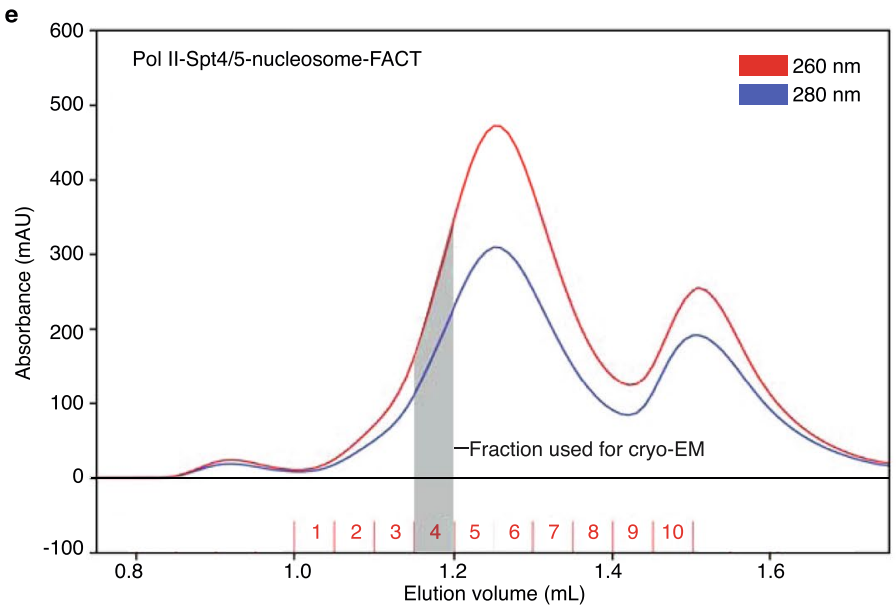

g

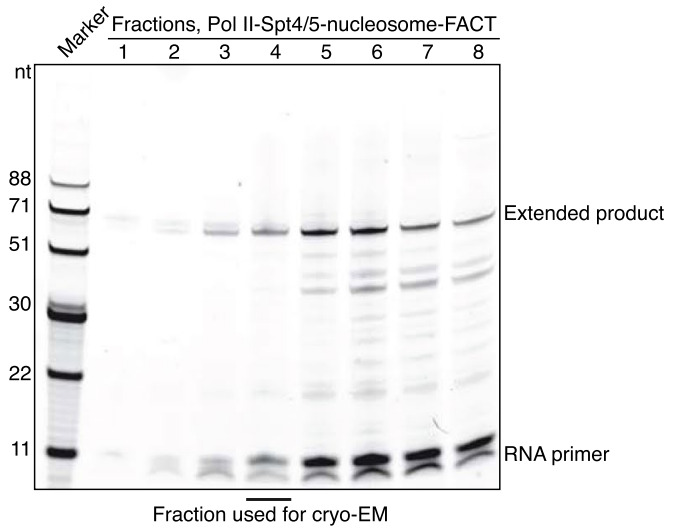

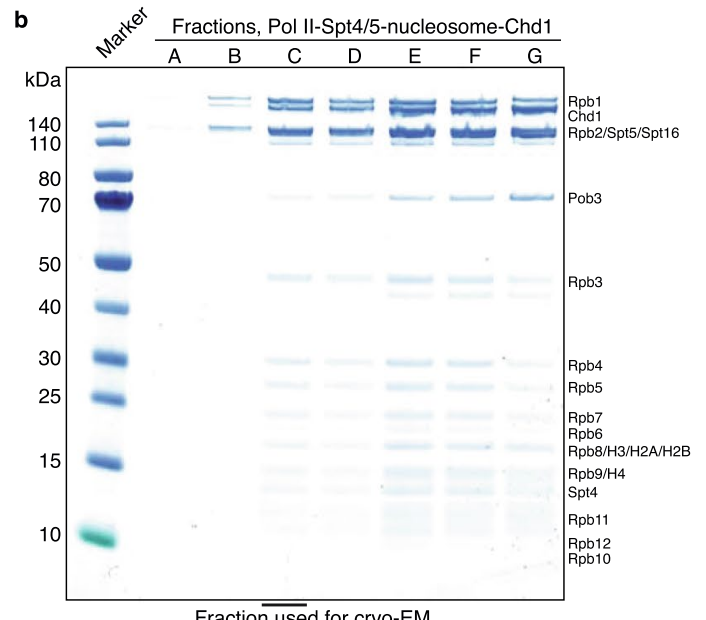
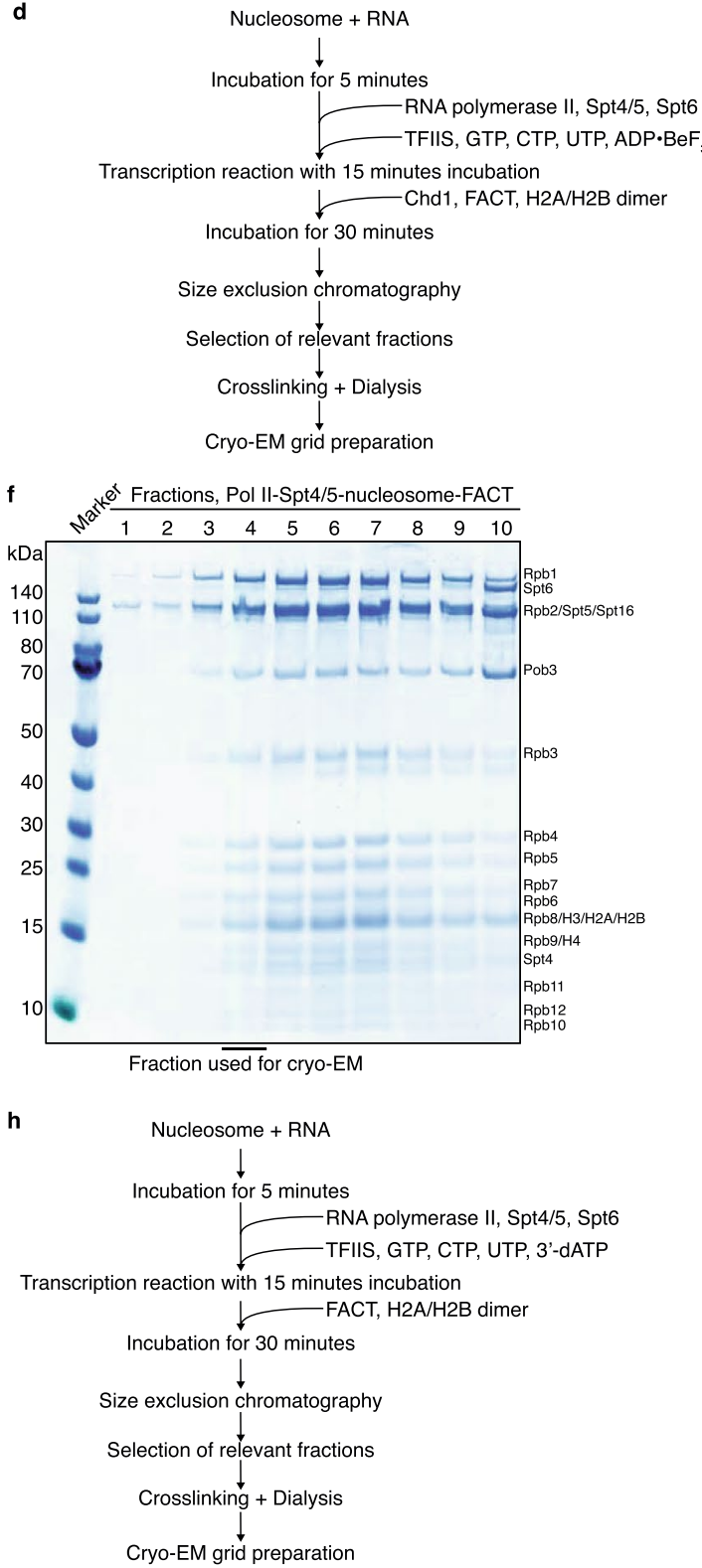

Extended Data Fig. 2 | See next page for caption. 
Extended Data Fig. 2 | Formation of Pol II-nucleosome complexes with bound Chd1 and FACT. a, Chromatogram of Pol II-Spt4/5-nucleosome-Chd1 complex formation using size exclusion chromatography. Fractions used in further analysis are indicated. b, SDS-PAGE of Pol II-Spt4/5-nucleosome-Chd1 complex formation. SDS-PAGE shows presence of FACT in the complex. c, $12 \%$ denaturing urea gel of Pol II-Spt4/5-nucleosome-Chd1 complex formation. RNA is visualized using 6-FAM label on 5' end of RNA. d, Step-by-step flowchart of complex formation for the Pol II-Spt4/5-nucleosome-Chd1 complex e, Chromatogram of Pol II-Spt4/5-nucleosome-FACT complex formation using size exclusion chromatography. f, SDS-PAGE of Pol II-Spt4/5-nucleosome-FACT complex formation. $\mathbf{g}$, 12 \% denaturing urea gel of Pol II-Spt4/5-nucleosome-FACT complex formation. RNA is visualized using 6-FAM label on 5' end of RNA. h, Step-by-step flowchart of complex formation for the Pol II-Spt4/5-nucleosome-FACT complex. Uncropped gel images for panels $\mathbf{b}, \mathbf{c}, \mathbf{f}, \mathbf{g}$ are provided in Source Data Extended Data Fig. 2. 


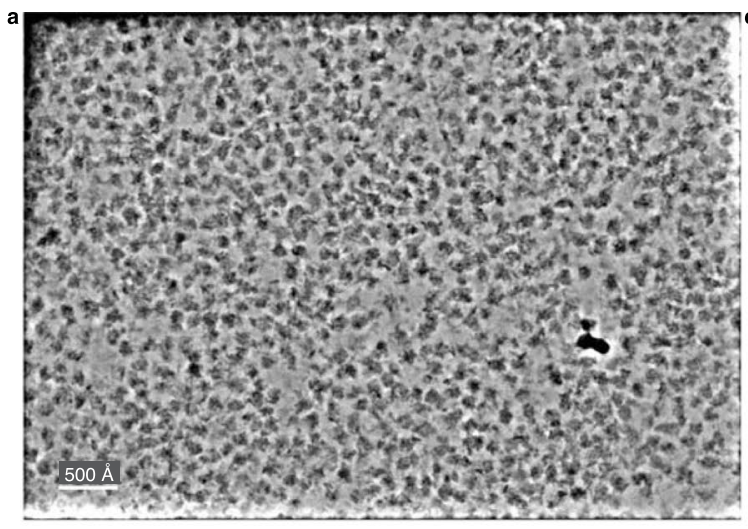

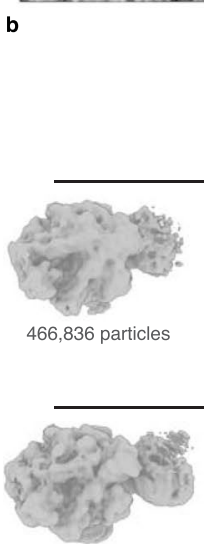

202,472 particles
$3,755,390$ BoxNet2 picked particles

Initial 3D classifications in cryoSPARC

I classification

Global 3D classificatio
$1,945,974$ particles

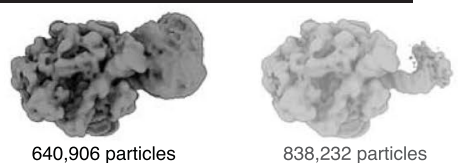

Global 3D classification

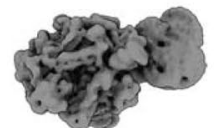

247,604 particles

Global 3D Refinement

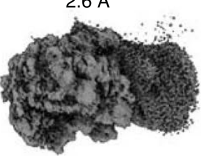

।

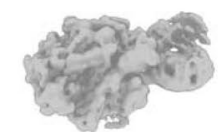

190,830 particles

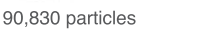

CTF refinement \& Bayesian polishing Masked classification 1 (Nucleosome-Chd1 mask)

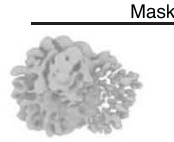

89,785 particles

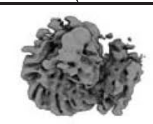

74,170 particles

e
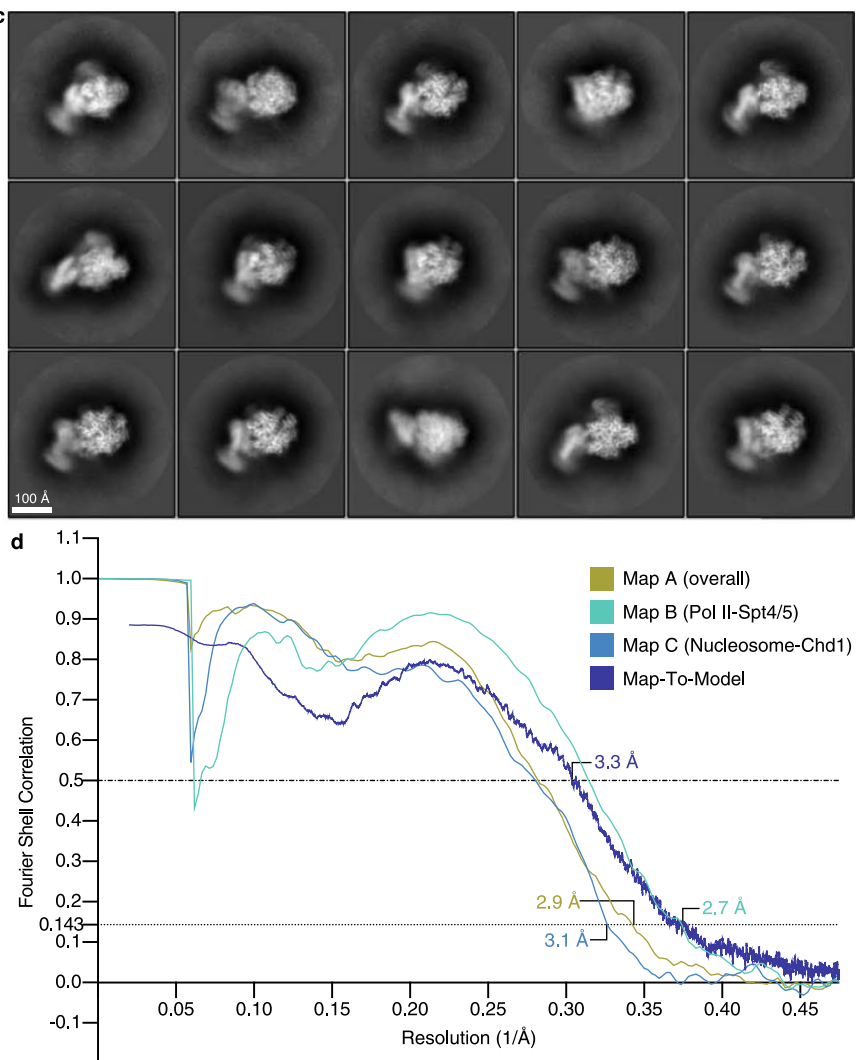

e
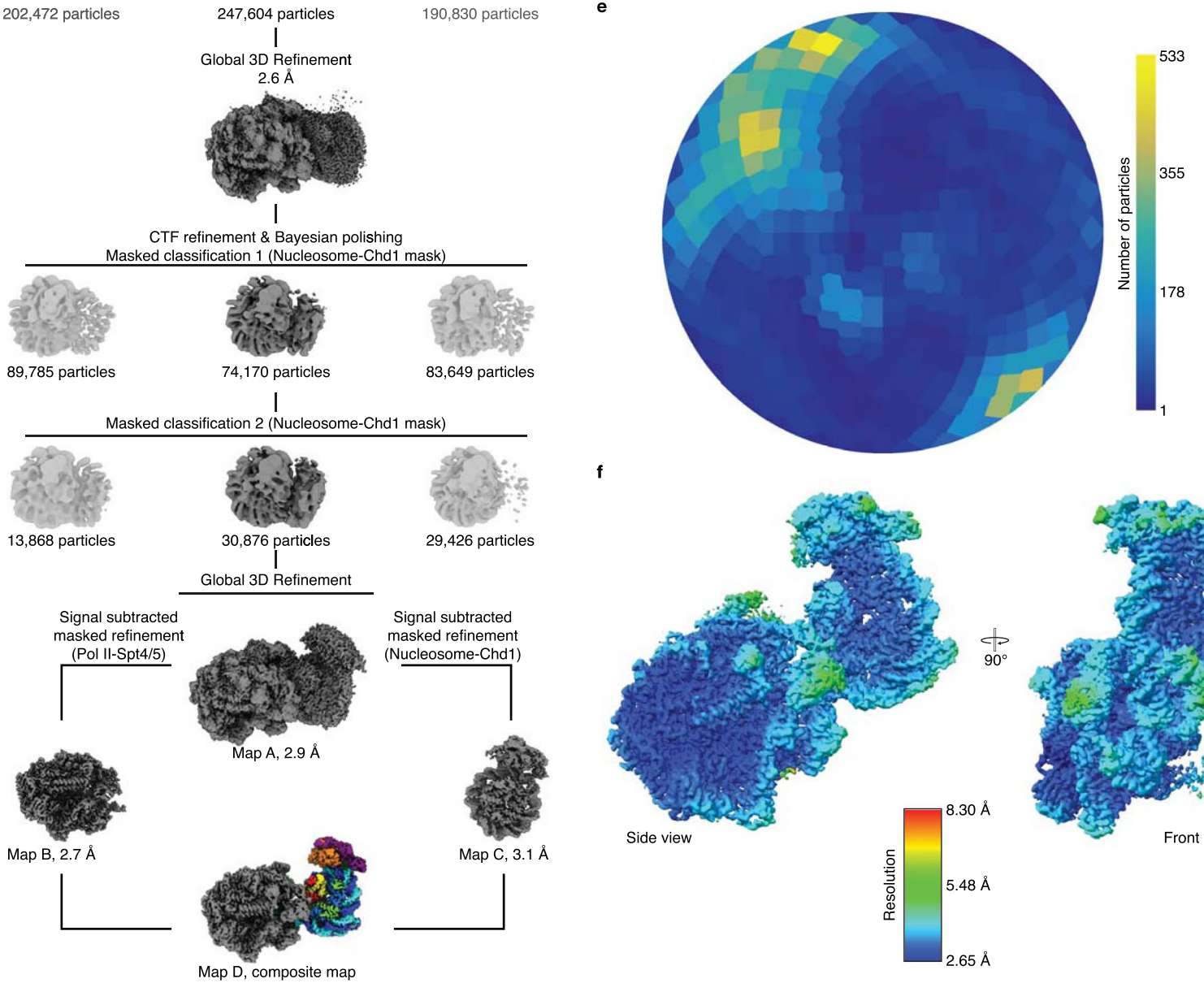

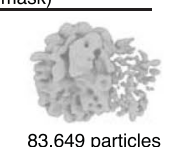

f

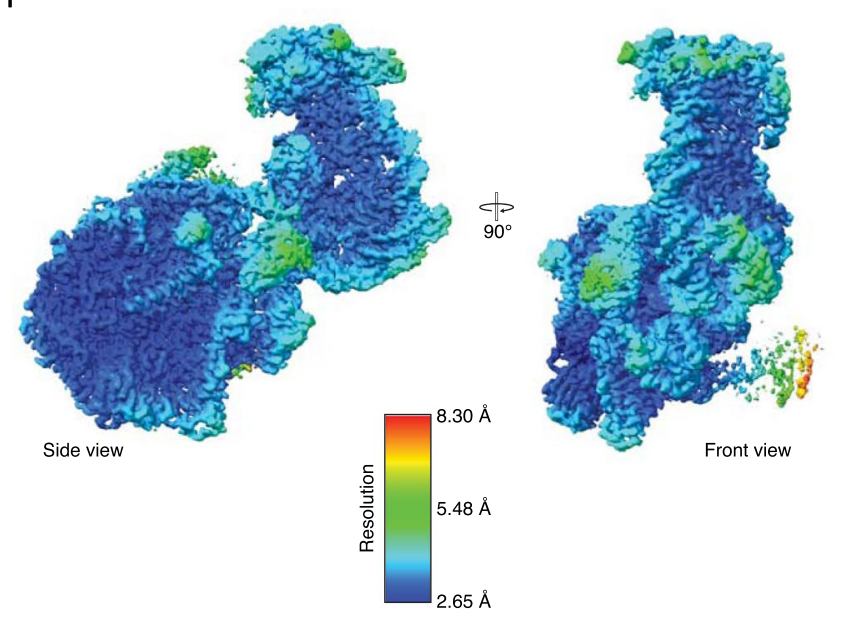

Extended Data Fig. 3 | See next page for caption. 
Extended Data Fig. 3 | Data acquisition, processing, and data quality metrics for the Pol II-Spt4/5-nucleosome-Chd1 structure. a, Representative denoised micrograph of data collection with scale bar $(50 \mathrm{~nm})$. b. Sorting and classification tree of Pol II-Spt4/5-nucleosome-Chd1 dataset. c, 2D classes of final refinement show RNA polymerase II and nucleosome-like shape with additional density (Chd1) with scale bar of $10 \mathrm{~nm}$. $\mathbf{d}$, FSC curves of maps A-C and map-to-model. Resolutions at FSC threshold criterions 0.143 or 0.5 are indicated. e, Angular distribution of particles employed to reconstruct map A. f, Local resolution of composite map D. 


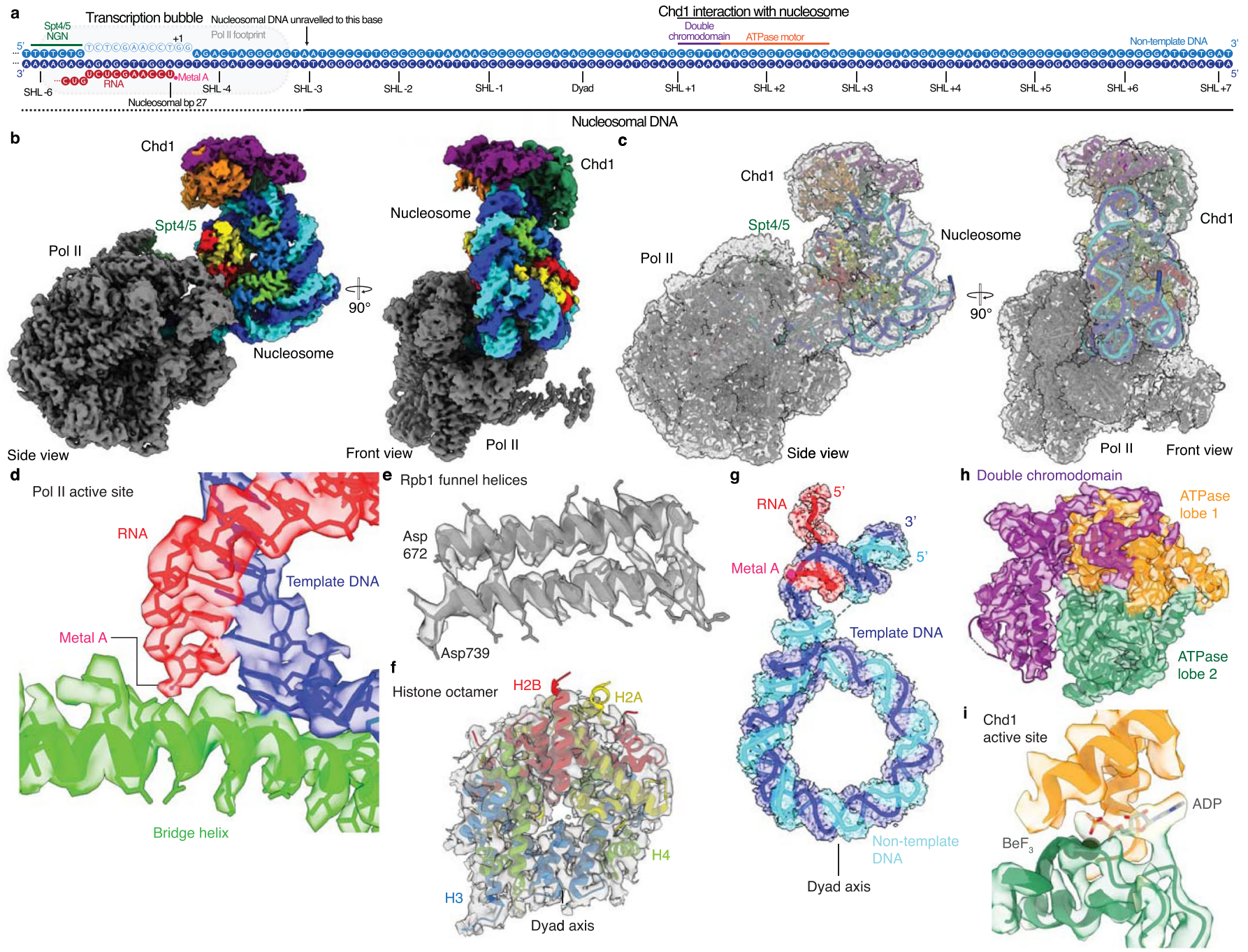

Extended Data Fig. 4 | Cryo-EM densities of Pol II-Spt4/5-nucleosome-Chd1 complex. a, Protein-nucleosomal DNA contacts of Pol II-Spt4/5-nucleosomeChd1 complex. Nucleotides are depicted as solid spheres (modelled) or empty spheres (not modelled). SHLs are indicated. b, Cryo-EM map (map D) of Pol II-Spt4/5-nucleosome-Chd1 complex. c, Pol II- Spt4/5-nucleosome-Chd1 structure with corresponding cryo-EM map (map D). Cryo-EM map is shown in grey. $\mathbf{d}$, Active site of Pol II-Spt4/5-nucleosome-Chd1 structure with corresponding density (map D). Metal A is shown as a pink sphere. e, Rpb1 funnel helices with corresponding density (map D). f, Histone octamer with corresponding density (map D). g, Nucleic acids in Pol II-Spt4/5-nucleosome-Chd1 structure with corresponding densities (map D) h, Chd1 with corresponding density (map D). i, Active site of Chd1 with bound ADP.BeF3. ADP is shown in stick representation, $\mathrm{BeF}_{3}$ as green spheres. Density from map D. 
a
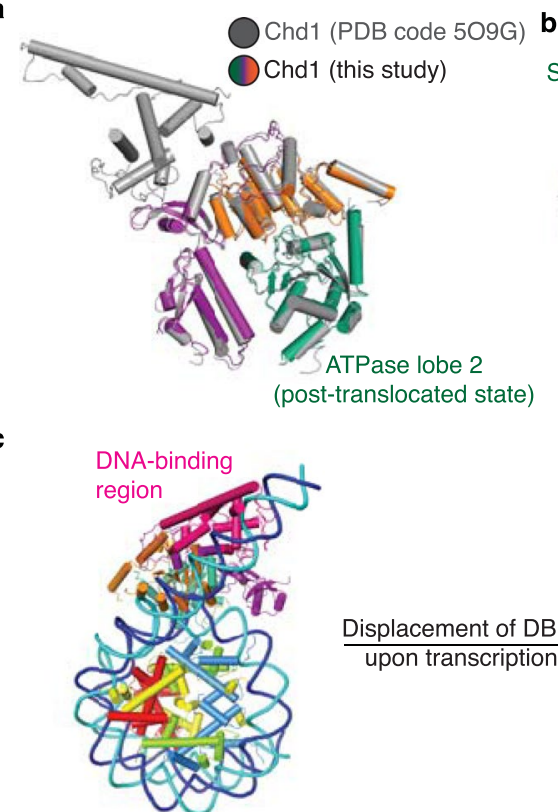

Nucleosome-Chd1 complex

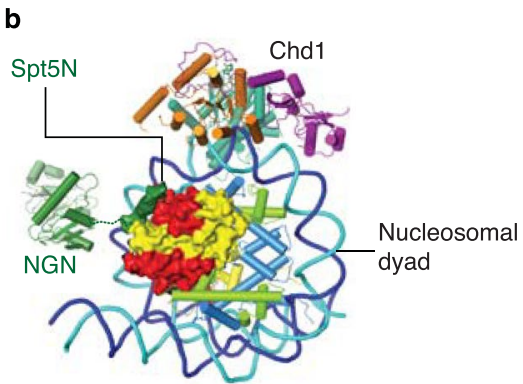

Unwrapped DNA due to transcription $\underset{\text { Displacement of DBR }}{\stackrel{\text { upon transcription }}{\longrightarrow}}$

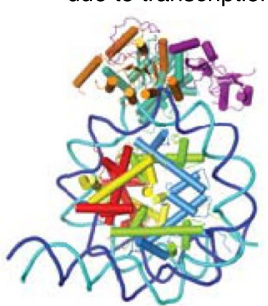

Pol II-Spt4/5-nucleosome-Chd1 complex

Extended Data Fig. 5 | Details of the Pol II-Spt4/5-nucleosome-Chd1 structure. a, Comparison of Chd1 (grey, PDB code 5O9G) with Chd1 (this study). The ATPase motor adopts the post-translocated state in both structures. b. Density for Spt5 N-terminal region (Spt5N) (low-passed filtered to $9 \AA$, map D) next to the proximal $\mathrm{H} 2 \mathrm{~A}-\mathrm{H} 2 \mathrm{~B}$ dimer (surface representation). c, Comparison of a poised nucleosome-Chd1 structure ${ }^{26}$ with the structure of $\mathrm{Chd1}$ bound to the transcribed nucleosome reveals displacement of the Chd1 DNA-binding region (DBR, pink) upon transcription. 

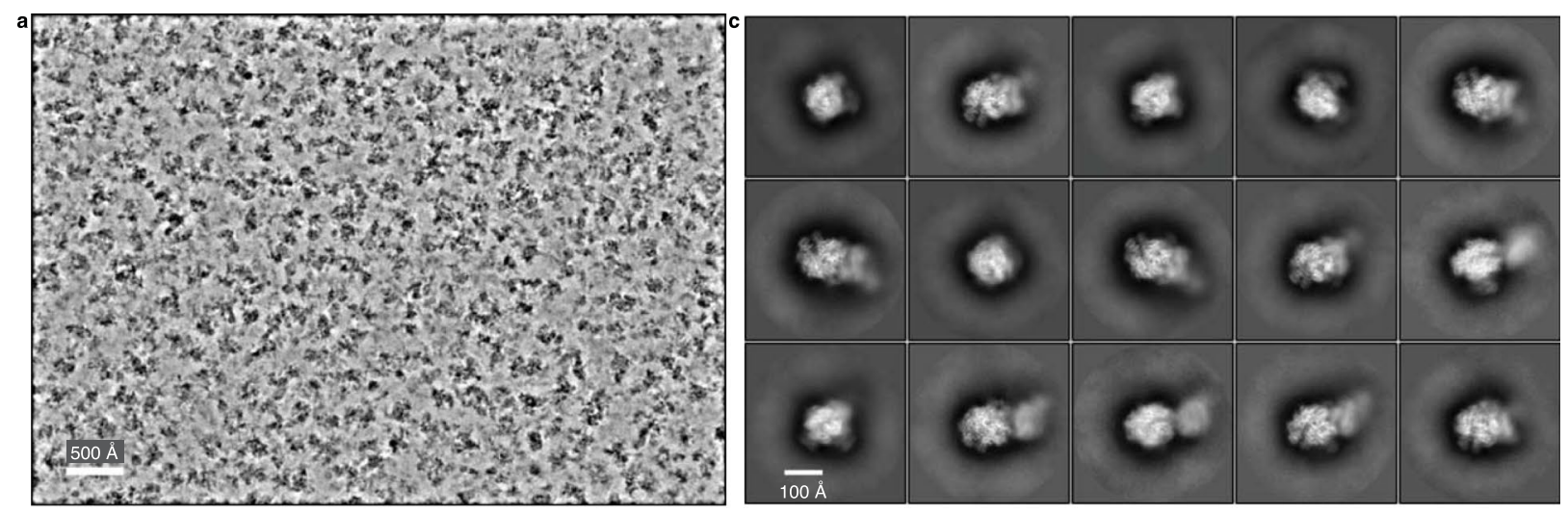

b

$5,227,093$ BoxNet2 picked particles

Initial 2D and 3D classification in cryoSPARC \& RELION |

Global refinement with $7.5^{\circ}$ sampling only 603,550 particles

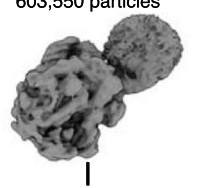

Masked classification (Nucleosome-FACT mask)

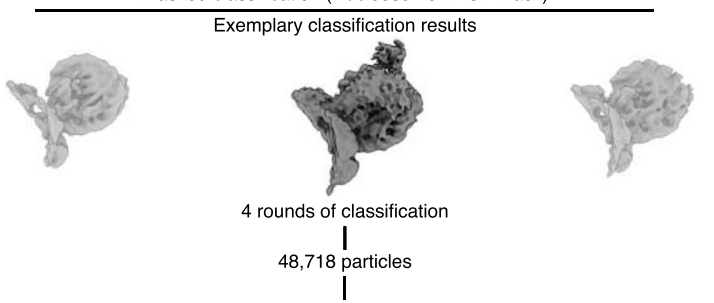

Masked classification (FACT mask)

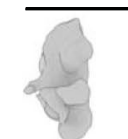

157 particles

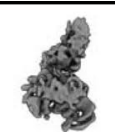

47,138 particles

38 partict

Global 3D Refinement

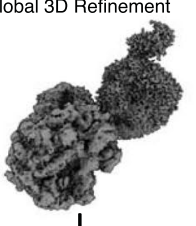

CTF refinement \& Bayesian Polishing

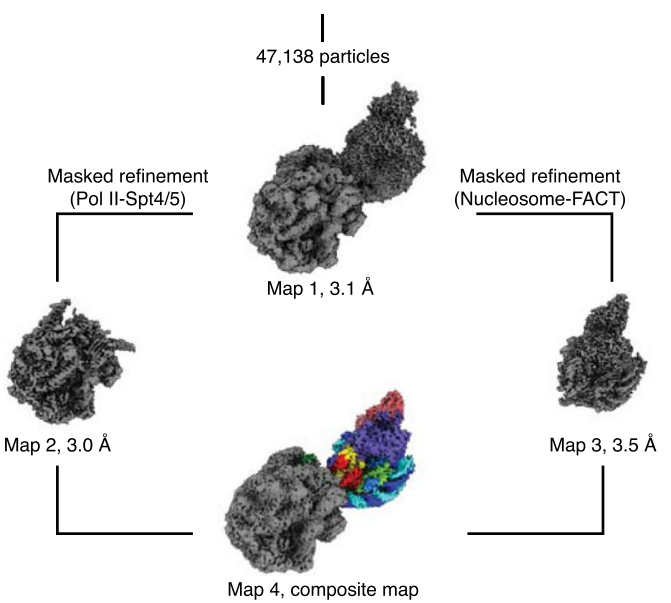

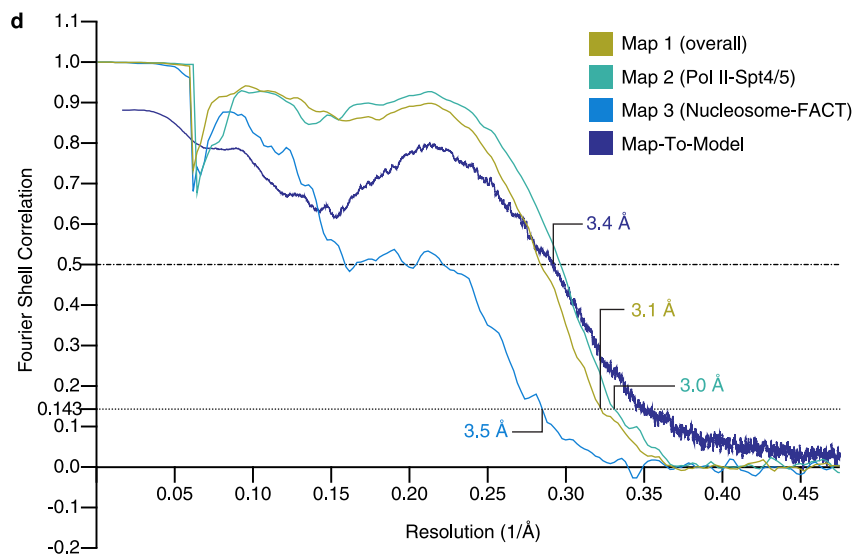
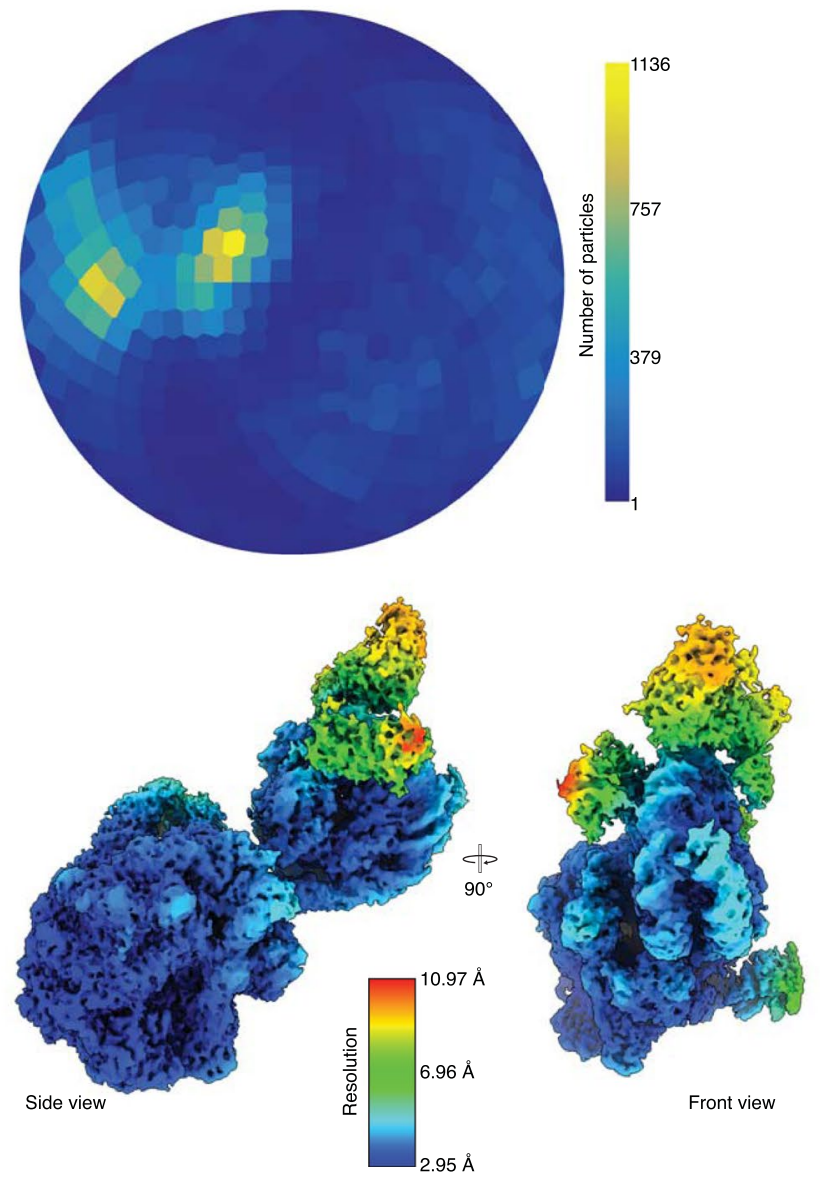

Extended Data Fig. 6 | See next page for caption. 
Extended Data Fig. 6 | Data acquisition, processing, and data quality metrics for the Pol II-Spt4/5-nucleosome-FACT structure. a, Representative denoised micrograph of data collection with scale bar $(50 \mathrm{~nm})$. b, Sorting and classification tree of Pol II-Spt4/5-nucleosome-FACT dataset. c, 2D classes of final refinement show RNA polymerase II and nucleosome-like shape with additional density (FACT) with scale bar of $10 \mathrm{~nm}$. $\mathbf{d}$, FSC curves of maps 1-4 and map-to-model. Resolutions at FSC threshold criterions 0.143 or 0.5 are indicated. e, Angular distribution of particles employed to reconstruct map 1. f, Local resolution of composite map 4. 

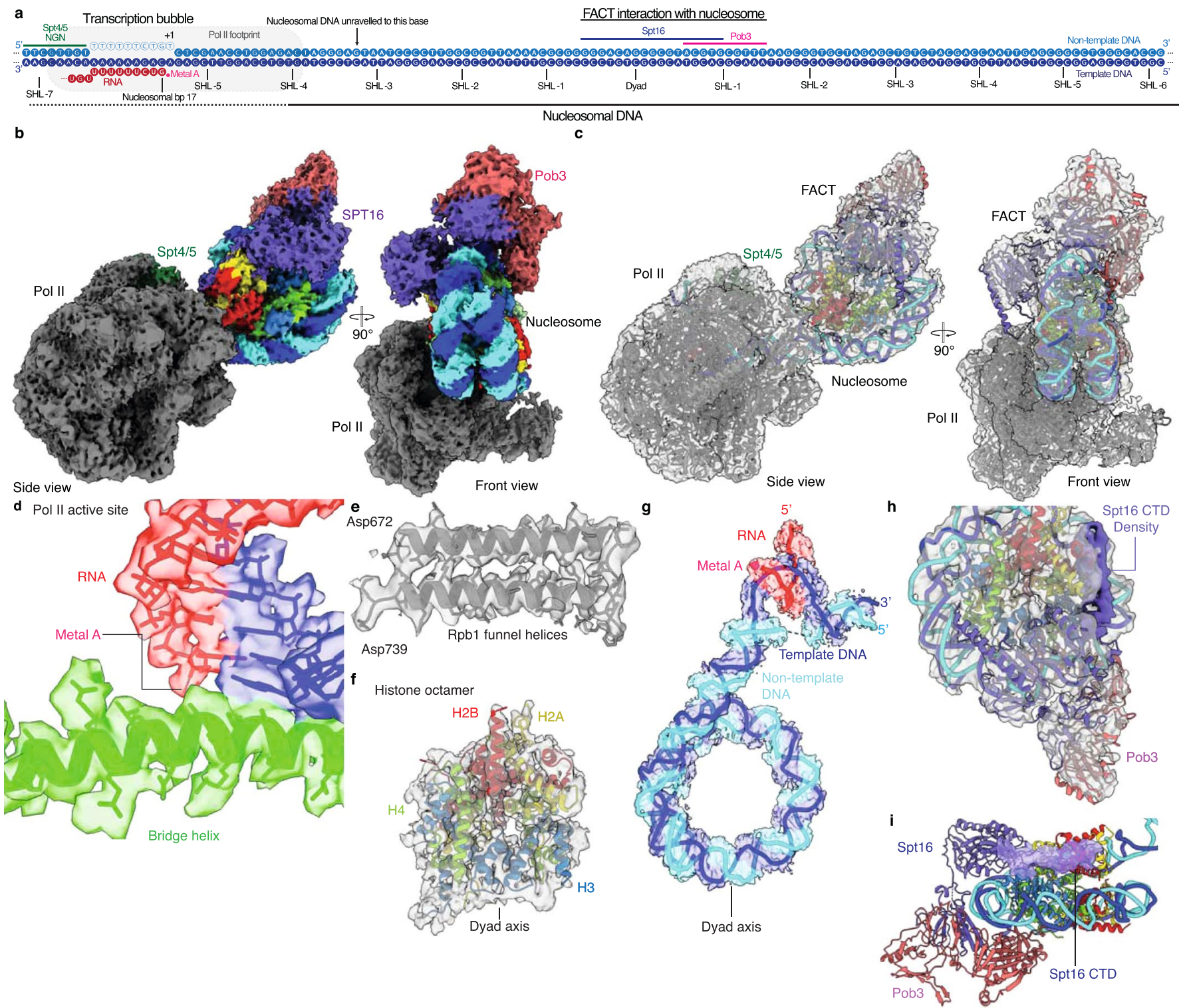

Extended Data Fig. 7 | Cryo-EM densities of Pol II-Spt4/5-nucleosome-FACT complex. a, Protein-nucleosomal DNA contacts of Pol II-Spt4/5-nucleosomeFACT complex. Nucleotides are depicted as solid spheres (modelled) or empty spheres (not modelled). SHLs are indicated. b, Cryo-EM map 4 of Pol II-Spt4/5-nucleosome-FACT complex. c, Pol II-Spt4/5-nucleosome-FACT structure with corresponding cryo-EM map (map 4). Cryo-EM map is shown in grey. $\mathbf{d}$, Active site of Pol II-Spt4/5-nucleosome-Chd1 structure with corresponding density (map 4). e, Rpb1 funnel helices with corresponding density (map 4).f, Histone octamer with corresponding density (map 4). g, Nucleic acids in Pol II-Spt4/5-nucleosome-FACT structure with corresponding densities (map 4). h, Nucleosome with bound FACT and corresponding density (map 4). Density corresponding to the Spt16 CTD is highlighted in purple. i, Spt16 CTD density (map4) contacts the proximal H2A/H2B dimer. 
a

H. sapiens FACT (PDB code 6UPL)

S. cerevisiae FACT (this study)

Nucleosome
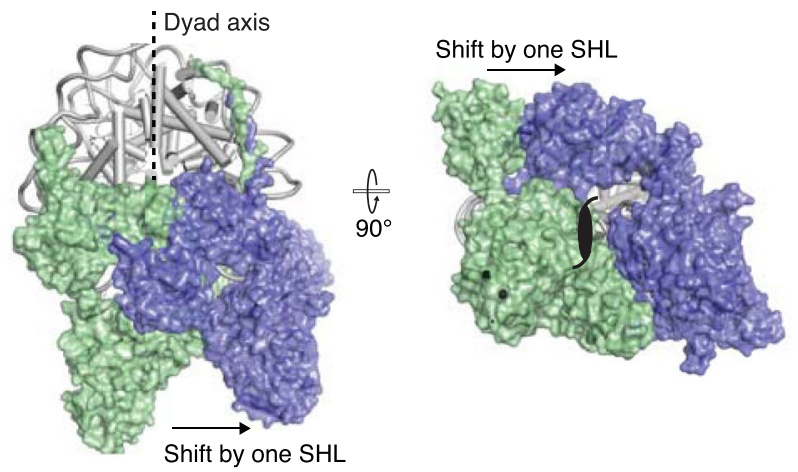

b

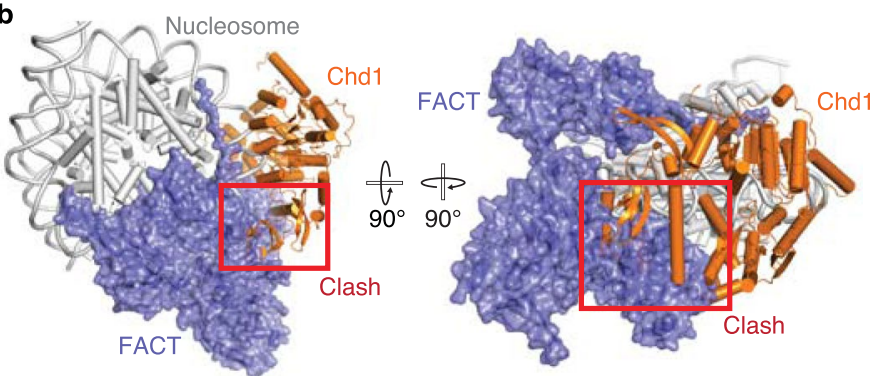

Extended Data Fig. 8 | Details of the Pol II-Spt4/5-nucleosome-FACT structure. a, Superposition of a subnucleosome-FACT complex (PDB code 6UPL; FACT, pale green) on the Pol II-Spt4/5-nucleosome FACT structures reveals sliding of FACT by one superhelical location. FACT-transcribed nucleosome and the subnucleosome structure were aligned using the histone octamer. Transcribed nucleosome is shown in grey. b, Superposition of Chd1 structure with the FACT structure reveals a steric clash between the ATPase lobe 2 and double chromodomain of Chd1 and the Pob3 subunit of FACT. 


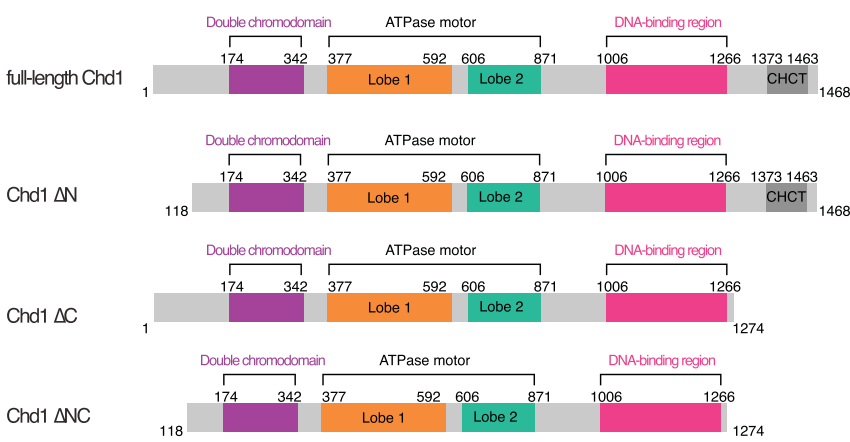

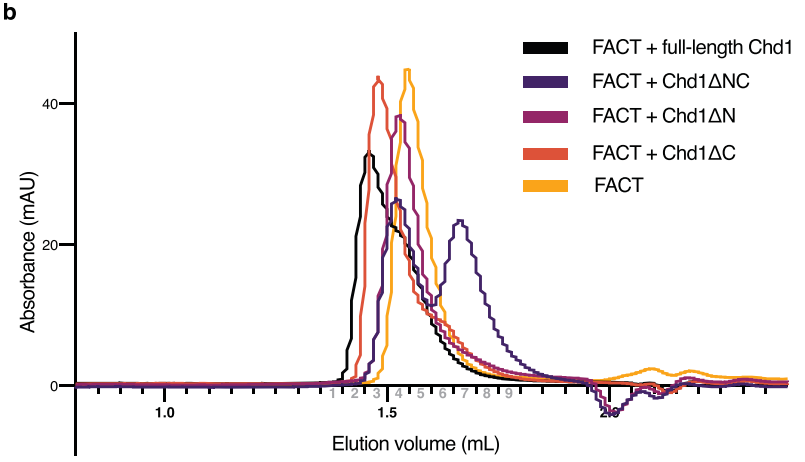
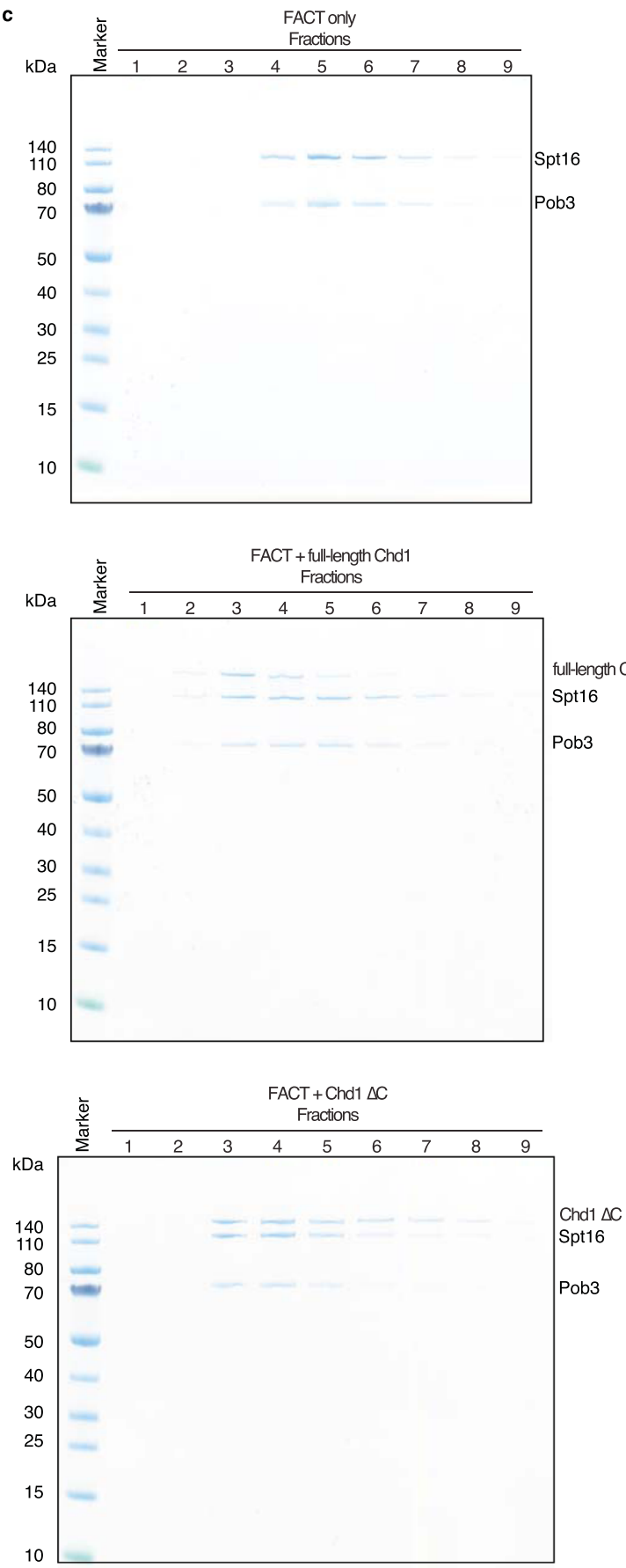
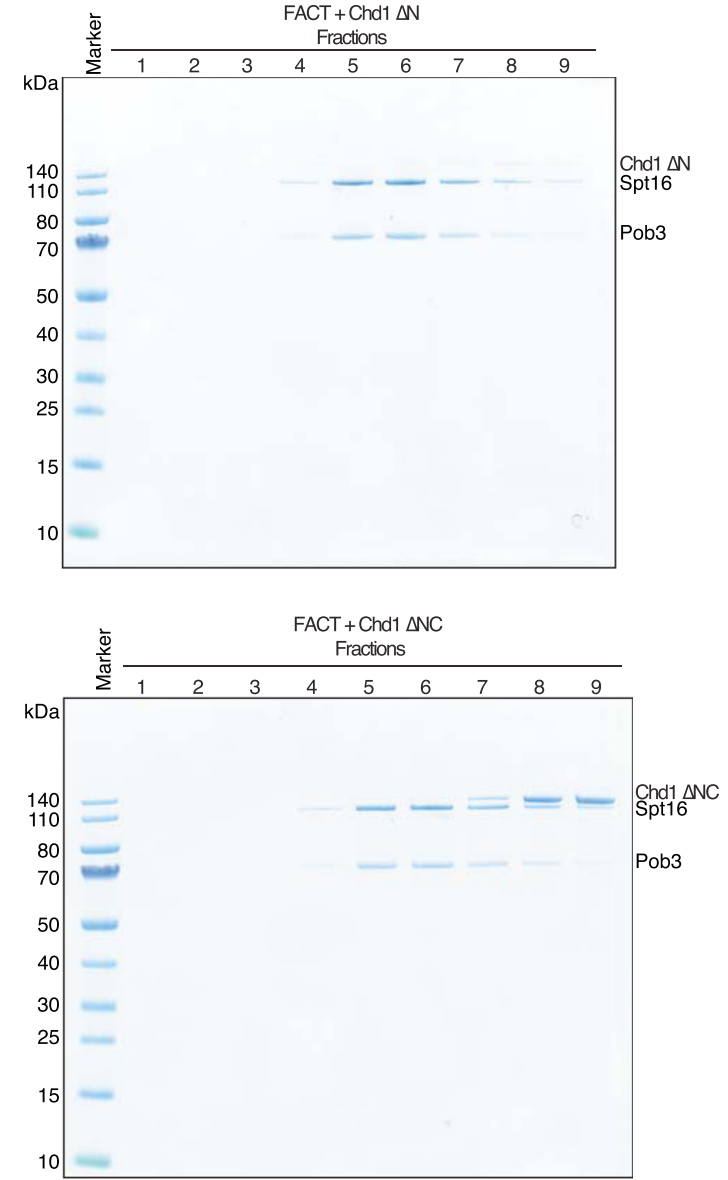

d Summary of Interaction with FACT interactions full-length Chd1 $\quad$ Ye Chd $1 \Delta \mathrm{N}$ Chd1 $\triangle C$ Chd1 $\triangle \mathrm{NC}$
Chd1 $\Delta N$ Pob3 
Extended Data Fig. 9 | Interaction between Chd1 and FACT. a, Domain architecture with different Chd1 constructs; full-length Chd1, Chd1 $\Delta \mathrm{N}$ (residues 118-1468), Chd1 $\Delta C$ (residues 1-1274) and Chd1 $\Delta N C$ (residues 118-1274). b. Chromatogram of size exclusion chromatography runs to determine regions of Chd1 that interact with FACT. Fractions analysed with SDS-PAGE (c) are indicated with grey numbering. c, SDS-PAGE analysis of size exclusion chromatography runs (b) reveals interaction of Chd1 with FACT via the N-terminus of Chd1. Uncropped gel images are available as Source Data Extended Data Fig. 9. d, Summary of interaction results. 
a Pol II approaching the nucleosome

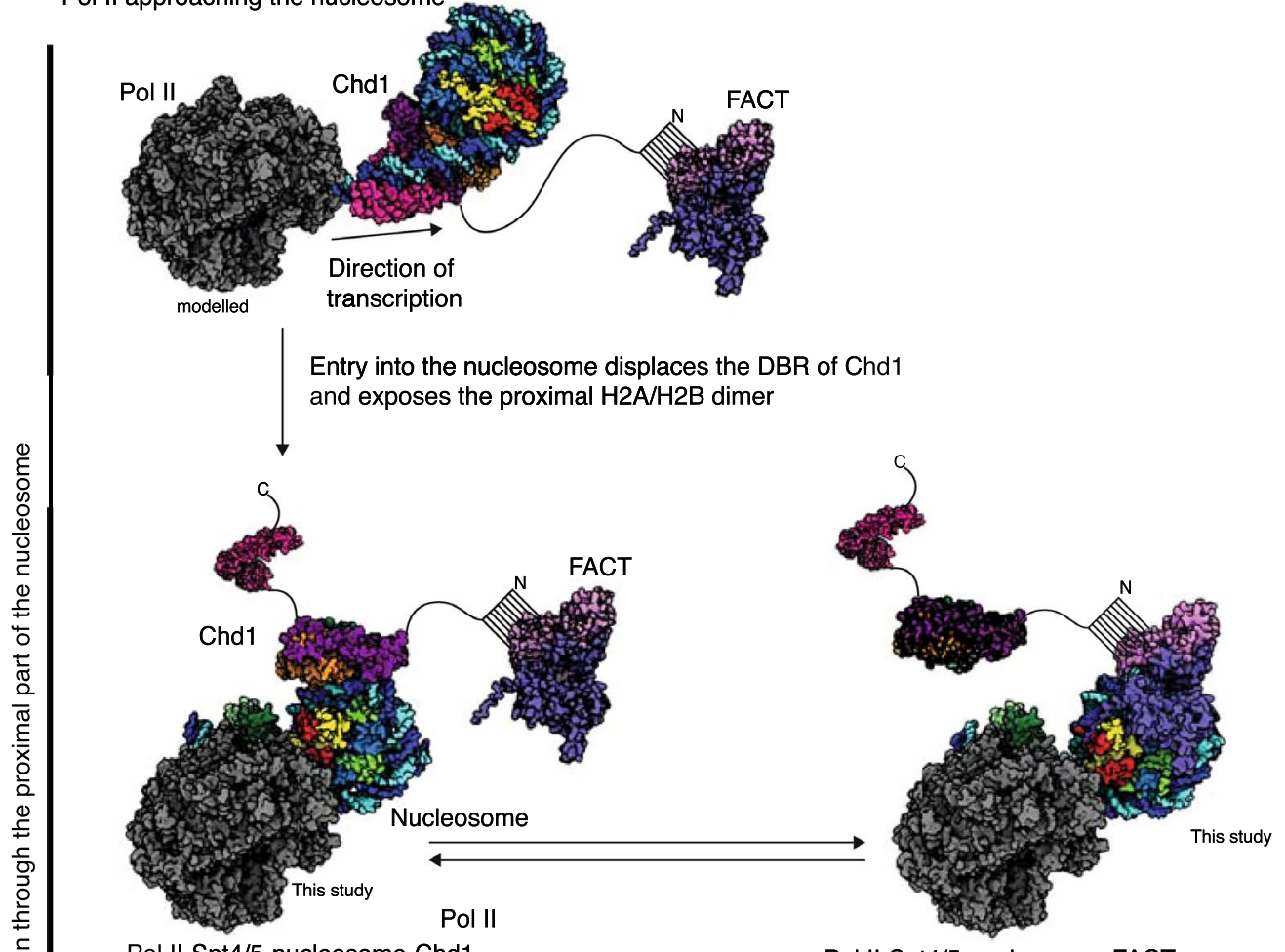

Pol II-Spt4/5-nucleosome-Chd1

Pol II-Spt4/5-nucleosome-FACT

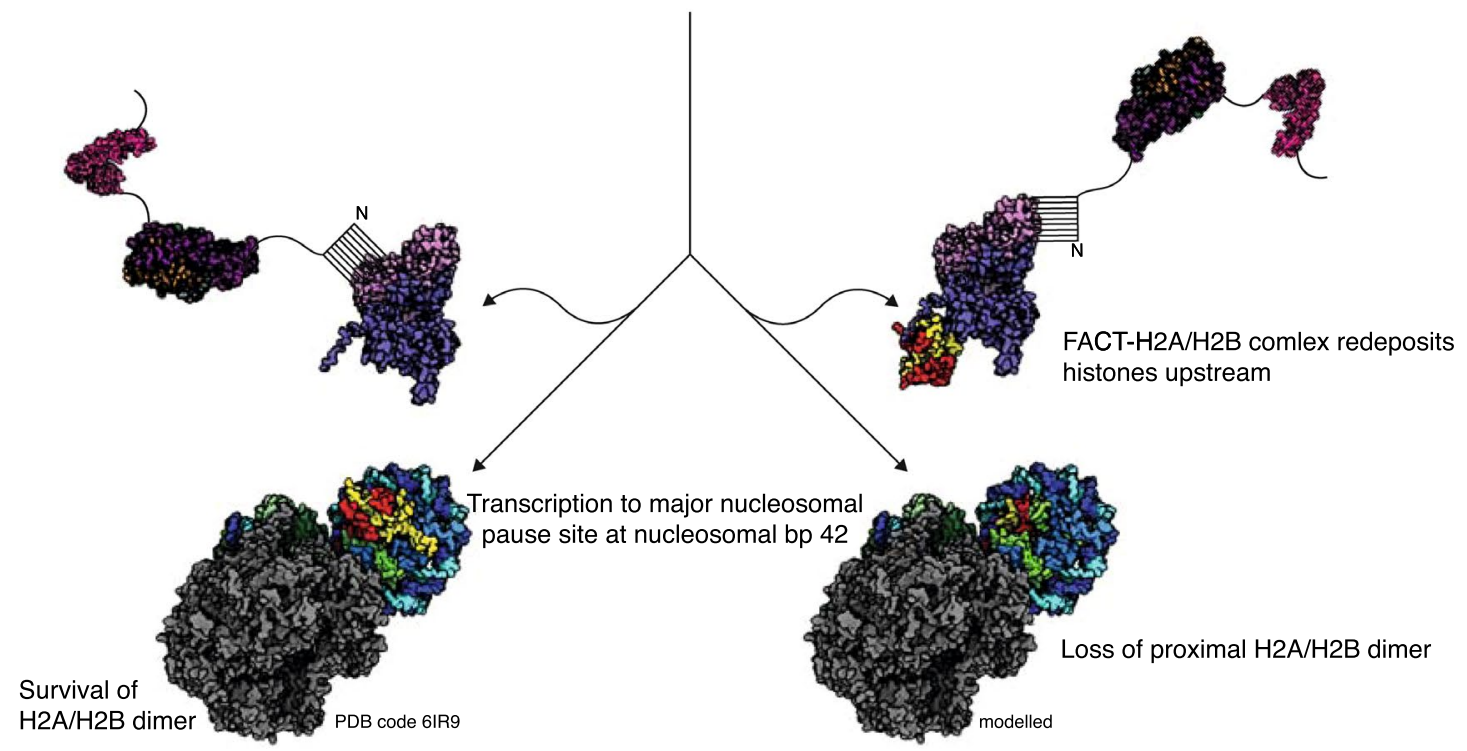

b

Progression of transcription to nucleosomal bp 17 Progression of transcription to nucleosomal bp 42

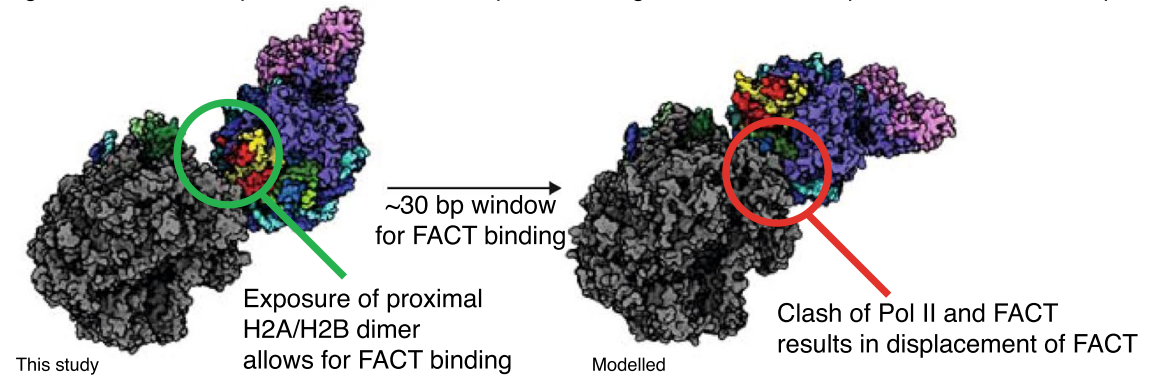

Extended Data Fig. 10 | Extended model for Pol II passage through a nucleosome. a, Extended model for Pol II progression through the proximal part of a nucleosomal substrate. $\mathbf{b}$, Structural modelling reveals a 30 bp window for FACT binding during transcription through the proximal part of the nucleosomal substrate. 


\section{Reporting Summary}

Nature Research wishes to improve the reproducibility of the work that we publish. This form provides structure for consistency and transparency in reporting. For further information on Nature Research policies, see our Editorial Policies and the Editorial Policy Checklist.

\section{Statistics}

For all statistical analyses, confirm that the following items are present in the figure legend, table legend, main text, or Methods section.

$\mathrm{n} / \mathrm{a}$ Confirmed

$\bigotimes$ The exact sample size $(n)$ for each experimental group/condition, given as a discrete number and unit of measurement

$\square$ A statement on whether measurements were taken from distinct samples or whether the same sample was measured repeatedly

The statistical test(s) used AND whether they are one- or two-sided

Only common tests should be described solely by name; describe more complex techniques in the Methods section.

$\bigotimes \square$ A description of all covariates tested

$\square$ A description of any assumptions or corrections, such as tests of normality and adjustment for multiple comparisons

$\square$ A full description of the statistical parameters including central tendency (e.g. means) or other basic estimates (e.g. regression coefficient)

AND variation (e.g. standard deviation) or associated estimates of uncertainty (e.g. confidence intervals)

For null hypothesis testing, the test statistic (e.g. $F, t, r$ ) with confidence intervals, effect sizes, degrees of freedom and $P$ value noted

$\triangle$ Give P values as exact values whenever suitable.

Х $\square$ For Bayesian analysis, information on the choice of priors and Markov chain Monte Carlo settings

Х $\square$ For hierarchical and complex designs, identification of the appropriate level for tests and full reporting of outcomes

Х $\square$ Estimates of effect sizes (e.g. Cohen's d, Pearson's $r$ ), indicating how they were calculated

Our web collection on statistics for biologists contains articles on many of the points above.

\section{Software and code}

Policy information about availability of computer code

Data collection Serial EM 3.8 beta 8

Data analysis RELION 3.0.17, UCSF Chimera 1.13, UCSF ChimeraX v1.11, Pymol 2.3.4, Coot 0.9, Warp v1.0.7-1.0.9, PHENIX 1.18, cryoSPARC 2.14.2

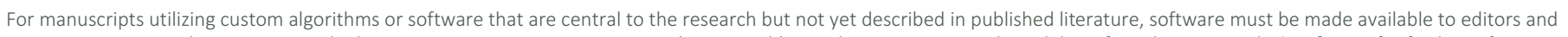

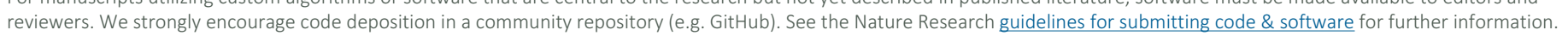

\section{Data}

Policy information about availability of data

All manuscripts must include a data availability statement. This statement should provide the following information, where applicable:

- Accession codes, unique identifiers, or web links for publicly available datasets

- A list of figures that have associated raw data

- A description of any restrictions on data availability

The cryo-EM reconstructions and final models for the Pol II-Spt4/5-nucleosome-Chd1 complex were deposited with the Electron Microscopy Data Base (EMD-12449) and the Protein Data Bank (PDB 7NKX). The cryo-EM reconstructions and final models for the Pol II-Spt4/5-nucleosome-FACT complex were deposited with the Electron Microscopy Data Base (EMD-12450) and with the Protein Data Bank (PDB 7NKY). For the Pol II-Spt4/5-nucleosome-Chd1 complex, maps A-C were deposited as EMD-12666, EMD-12667 and EMD-12668, respectively. For the Pol II-Spt4/5-nucleosome-FACT complex, maps 1-3 were deposited as EMD-12669, EMD-12670 and EMD-12671, respectively. Source data are provided with this paper. 
Please select the one below that is the best fit for your research. If you are not sure, read the appropriate sections before making your selection.

$\bigotimes$ Life sciences

Behavioural \& social sciences

$\square$ Ecological, evolutionary \& environmental sciences

For a reference copy of the document with all sections, see nature.com/documents/nr-reporting-summary-flat.pdf

\section{Life sciences study design}

All studies must disclose on these points even when the disclosure is negative.

\begin{tabular}{|c|c|}
\hline Sample size & No statistical methods were used to predetermine sample size. \\
\hline Data exclusions & No data were excluded from the analyses. \\
\hline Replication & $\begin{array}{l}\text { All attempts at replication were successful. Cryo-EM single particle analysis inherently relies on averaging over a large number of independent } \\
\text { observations. }\end{array}$ \\
\hline Randomization & Samples were not allocated to groups. \\
\hline Blinding & Investigators were not blinded during data acquisition and analysis because it is not a common procedure for the methods employed. \\
\hline
\end{tabular}

\section{Reporting for specific materials, systems and methods}

We require information from authors about some types of materials, experimental systems and methods used in many studies. Here, indicate whether each material, system or method listed is relevant to your study. If you are not sure if a list item applies to your research, read the appropriate section before selecting a response.

\begin{tabular}{|c|c|c|c|}
\hline \multicolumn{2}{|r|}{ Materials \& experimental systems } & \multicolumn{2}{|c|}{ Methods } \\
\hline$n / a$ & Involved in the study & $\mathrm{n} / \mathrm{a}$ & Involved in the study \\
\hline$\bigotimes$ & $\square$ Antibodies & Х & ChIP-seq \\
\hline $\mathbf{L}^{\mathrm{a}}$ & $\bigotimes$ Eukaryotic cell lines & Х & $\square$ Flow cytometry \\
\hline$\bigotimes$ & $\square$ Palaeontology and archaeology & Х & $\square$ MRI-based neuroimaging \\
\hline$\bigotimes$ & Animals and other organisms & & \\
\hline$\bigotimes$ & Human research participants & & \\
\hline$\bigotimes$ & Clinical data & & \\
\hline$凶$ & $\square$ Dual use research of concern & & \\
\hline
\end{tabular}

\section{Eukaryotic cell lines}

Policy information about cell lines

Cell line source(s)

Authentication

Mycoplasma contamination

Commonly misidentified lines (See ICLAC register)
Hi5 cells: Expression Systems, Tni Insect cells in ESF921 media, item 94-002F

Sf9 cells: ThermoFisher, Catalogue Number 12659017, Sf9 cells in Sf-9000TM III SFM Sf21 cells: Expression Systems, SF21 insect cells in ESF921 medium, Item 94-003F

None of the cell lines were authenticated.

Cell lines were not tested for mycoplasma contamination.

No commonly misidentified cell lines were used. 